\title{
Modern rheology and electric induction: multivalued index of refraction, splitting of eigenvalues and fatigue
}

\author{
Michele Caputo \\ Dipartimento di Fisica, Università «La Sapienza», Roma, Italy
}

\begin{abstract}
The modern constitutive equations of the rheology of anelastic media and of the polarization in dielectric media are discussed in the time and frequency domains. It is seen that the most appropriate form of the constitutive equations of these media is based on the convolution of the stress and strain, and of the electric field and induction respectively, with appropriate functions representing the memory of the medium to previous stress or induction. The most successful memory formalism in representing the observed phenomena is that of the derivative of fractional order which allows a variety of problems to be solved by means of the Laplace Transform. The consequent index of refraction, generally, is a set valued function which implies that a monochromatic wave generated in the medium is split into a set of waves with the same frequency but different wavelengths which interfere and have a quasi periodically varying amplitude. The eigenfunctions of these media, associated to the ordinarily used principal values of the index of refraction, are split and so are the free modes. The fatigue of anelastic media is studied using the new constitutive equations containing fractional derivatives; a phenomenological method is introduced to compute the number of cycles which gives fatigue for a strain or stress with given amplitude and frequency. The number of cycles which may cause fatigue is proportional to the amplitude and frequency of the applied strain or stress, or inversely proportional to the rate of stress or strain. A criterion is also established to see whether an applied cyclic stress or strain may cause fatigue. This criterion is tested with experimental data on steel and rocks verifying that, in general, it is consistent with the data although the data are not always in the linear range. It is tentatively seen that fatigue does not seem one of the causes of the release of the elastic energy stored in the Earth.
\end{abstract}

Key words rheology - induced polarization refraction index - eigenvalues splitting - fatigue

\section{Introduction}

Because of gravity mountain ranges may sink or rebound in the Earth's crust, glaciers and land slide down the valley adapting to the shape of the topography and in turn shaping it, sediments compact in the surface layers and

Mailing address: Michele Caputo, Dipartimento di Fisica, Università «La Sapienza», Piazzale Aldo Moro 2, 00185 Roma, Italy; e-mail: mcaputo@axcasp.caspur.it also in the interior of the mantle when gravity brings them down with the continental slabs.

Gravity moves the center of gravity of the Earth regularly within the Earth itself because of the rotation of the Earth's bulges. In fact, these have revolution symmetry with respect to the axis connecting the centers of gravitation of the two attracting bodies but are not symmetric with respect to the center of gravity of the Earth; since they rotate relative to the body of the Earth, the center of gravity follows the rotation. Moreover the Moon and the Sun are not in the equatorial plane of the Earth and have different orbital planes, then the center of gravity of the Earth describes a non closed and non planar line within the Earth itself. 
The effect of gravity is dominant not only in the dynamics of the stars and planetary systems but also in the long term evolution of the universe since it causes the gravitational collapse of the stars by means of which they become neutron stars or give origin to supernovae.

However, in the intermediate term evolution of the universe, the dissipation of energy, through anelastic phenomena, is also important.

An example very close to us is that of the evolution of the Earth-Moon system. In fact, the semiaxes of the orbits of the two bodies change in time due to the action caused by the lag of the tidal bulges relative to the direction of the body causing them; eventually the two bodies, in the long run, will fall on each other.

The same is true for all the other planets of the solar system due to their tidal interaction with the sun and, for the same reason, for all the other planetary or binary systems of the universe.

Since the lag of the tidal bulges is determined by the rheology of the body, it is clear that the rheology of anelastic bodies contributes significantly to the intermediate evolution of all the planetary systems and of the universe.

Obviously the rheology and the consequent dissipation of elastic energy in the dynamic processes is also important in shaping the dynamics of the interior of the Earth, of many planets and of all stars.

It is therefore clear that the rheology of anelastic media contributes significantly to the intermediate term changes in the structure of the universe; and that this poorly known but very important phenomenon requires great attention, a much greater attention than that given in the past.

\section{Why do we need new relations between stress (applied electric field) and strain (induction)?}

The constitutive equations of the perfectly elastic bodies, that is the law of Hooke, and of the perfect dielectrics, that is the proportional- ity between the applied electric field and the induction, imply that when one sets oscillations in the medium, the oscillations will persist for an infinite time and the energy is not dissipated. Moreover the phase velocity of the waves does not depend on its frequency or on its wavelength; that is to say, when the medium is homogeneous, the product of the frequency of a wave times its wavelength is constant.

However it is well known that the elastic energy of all bodies decays in time and is in part transformed into heat, through the so called internal friction, in part used for the migration of crystal dislocation which, in turn, is again in part transformed into heat.

It is easily verified that the law of Hook is not satisfied when considering the bulges of the Earth caused by the tidal fields of the Moon and the Sun. In fact, the time variation of these fields is so slow that inertial forces may be neglected and the anelastic phenomena may be considered static. The stress strain relations of the Hooke type should represent the phenomena with sufficient accuracy since these fields cause deformations of the order of $10^{-8}$, which are generally accepted as linear. However it has been observed with sufficient accuracy (Slichter et al., 1964) that these bulges are not aligned with the body causing the tidal field but they lag (or in the Earth's reference system they lead) the position of the body causing the field. This clearly implies that the time variable must be present in the constitutive equations in order to allow for the phase difference between the effect and the cause.

The same considerations apply to solid, fluid and dielectric plasmas and is verified for instance in geoelectric prospecting when observing that a boxcar shaped electric field injected in the ground is evidently received at the recording instrument as a signal increasing at a decreasing rate and then decreasing to zero at a different decreasing rate.

In order to represent the above mentioned phenomena, as well as others, Hooke's constitutive equations of elasticity, as well as the classic proportionality relation between the electric field and the induction have, since long, 
been changed to introduce the appropriate time variable in these relations.

The simplest attempts at reshaping the law of Hooke in order to accomodate anelastic phenomena were those of Kelvin Voigt and of Maxwell who wrote the stress strain relations in the following form

$$
\begin{array}{ll}
\tau=(\alpha+\gamma \partial / \partial t) \varepsilon & \text { Kelvin-Voigt } \\
(\sigma+\eta \partial / \partial t) \tau=\gamma(\partial / \partial t) \varepsilon & \text { Maxvell }
\end{array}
$$

where $\tau$ and $\varepsilon$ are the stress and the strain respectively and $\alpha, \eta, \sigma, \gamma$ are parameters with the appropriate dimensions.

In electromagnetic phenomena, for instance in the study of the motion of the electronic cloud around the nucleus, which is the usual preliminary discussion made to introduce the study of dielectrics, Debye suggested a relation which may be written in the time domain in the following form

$$
\sigma \tau=(\alpha+\eta \partial / \partial t) \varepsilon
$$

where now $\varepsilon$ is the induction, $\tau$ is the applied electric field and $\alpha, \eta, \sigma$ are parameters with the appropriate dimensions.

Since the relations of Kelvin-Voigt, Maxwell and Debye soon proved inadequate to represent even the dissipation of energy of anelastic and dielectric media respectively, over relatively limited frequency ranges, it was necessary to consider more general relations between the stress and the strain or between the electric field and the induction.

In elasticity the generalization was first made extending the Maxwell or Kelvin-Voigt relations by adding one more derivative as follows

$$
(\sigma+\eta \partial / \partial t) \tau=(\alpha+\gamma \partial / \partial t) \varepsilon
$$

which, in anelasticty, is the so-called standard linear solid model.

However, the relation (2.4) was also shown inadequate to represent the rheology, the dissipation and the dispersion phenomena in anelastic media, and new more comprehensive forms than (2.4) were necessary to represent the above mentioned phenomena. The new forms, which contain memory formalisms, will be introduced and discussed in the following section.

\section{The constitutive memory relations of the anelastic and the dielectric media in 3D}

As already stated the introduction of the first order derivatives in the constitutive equations of elasticity, leading to the so-called Kelvin-Voigt or Maxwell or standard linear solids models, or the introduction of derivatives of higher order, were completely unsuccessful in explaining the decay of energy and dispersion.

In the first successful attempt at describing, with memory mechanisms, the $Q$ of many elastic materials, which is frequency independent (Knopoff and MacDonald, 1958) over wide frequency ranges, Caputo $(1966,1969)$ and Caputo and Mainardi (1971a) introduced the derivatives of fractional order in the onedimensional stress-strain relation. It was soon shown that these relations (Bagley and Torvik, 1983a,b, 1986) are causal and that they represent the decay of energy of a wide class of anelastic media.

In order to study the dispersion, the $Q$ of the shear waves and that of the purely dilatational waves it was necessary to generalize further the stress-strain relations and to consider them in three dimensions; for this purpose Caputo (1984) introduced in the relations more general operators which, when necessary, may be specialized in derivatives of fractional order.

In $3 \mathrm{D}$ it has been customary to assume that the quasi static purely dilatation phenomena have a negligible dissipation of energy and dispersion relative to those associated to the shear phenomena; therefore, the stress-strain relation in the time domain are, in general, written in order to satisfy this requirement as well.

However, rigourously, the assumption that the dilatation is perfectly elastic, even in quasi static phenomena, is a theoretical speculation which is valid only when the inertia forces are 
negligible and it may be presented as the asymptotic aspect of the dynamic case.

The stress-strain relations in $3 \mathrm{D}$ and the time domain, considering that there may be more than one dissipation and dispersion mechanisms represented by different memory functions, may therefore be written in a general form which separates the anelastic phenomena related to the dilatation from those related to shear and then specialized to the different limiting cases of interest.

Caputo (1984) wrote the constitutive equations in the following form

$h^{*} \tau_{i j}^{\prime}+\mu\left(\tau_{i j}-\tau_{r r} / 3\right)=2 m^{*} \varepsilon_{i j}^{\prime}+\delta_{i j} l^{*} \varepsilon_{r r}^{\prime}$

where $l(t)$ and $m(t)$ have dimension $\mathrm{gr}^{2} \mathrm{~cm}^{-2}$ $\mathrm{s}^{-3}, h(t)$ has the dimension $\mathrm{gr} \mathrm{cm}^{-1} \mathrm{~s}^{-1}$. There are three different memory mechanisms here, represented by the memory functions $l(t), m(t)$ and $h(t)$ whose Laplace Transform (LT) are indicated by $L(p), M(p)$ and $H(p)$ respectively with $p$ LT variable.

The equations of equilibrium, taking into account the stress-strain relations, may be written in the LT domain form by means of the following substitution

$$
\begin{aligned}
& \{\lambda\} \rightarrow[\mu(L / H+2 M / 3 H)+p L] /(p H+\mu) \\
& \{\mu\} \rightarrow p M /(p H+\mu) .
\end{aligned}
$$

In the LT domain the fourth order elastic tensor $c_{i j r s}$, represented by eq. (3.1), may be written

$$
\begin{aligned}
c_{i j r s}= & {[\mu(L / H+2 M / H)+p L] \delta_{i j} \delta_{r s} /(p H+\mu)+} \\
& +p M\left(\delta_{i r} \delta_{j s}+\delta_{i s} \delta_{j r}\right) /(p H+\mu) .
\end{aligned}
$$

From (3.1) and (3.2) it is seen that the effect of the memory mechanisms (3.1) on the pure dilatation is represented by

$$
T=T_{r r}=[(3 L+2 M) / H] \Delta
$$

where $\Delta$ is the LT of the dilatation and $T_{i j}$ are the LT of the stress components.
The effect of the memory mechanism on the shear is clearly given by the second of (3.2).

When $M$ and $L$ are proportional to $H$ then the dilatation is not affected by the memory mechanisms present in the stress-strain relations and, concerning the dilatation, everything happens as in perfect elasticity.

When $h=$ constant $=\eta$ then $H(p)=\eta / p$ and the effect of the memory functions is due to the combination $3 M+2 L$, which gives

$$
\begin{array}{ll}
T=p R \Delta & \text { or } \tau_{j j}=r^{\prime}(t)^{*} \varepsilon_{j j} \\
T_{i j}=2 p M E_{i j} /(\mu+\eta) & \text { or } \\
\tau_{i j} & =2 m^{\prime}(t)^{*} \varepsilon_{i j} /(\mu+\eta), i \neq j
\end{array}
$$

where we have set $R=3 M+2 L$ and $R(p)=$ $=\mathrm{LT}(r(t))$.

It is seen in (3.5a) that shear and dilatation memory are independent and represented by the independent functions $r(t)$ and $m(t)$. In fact, due to the presence of $l(t)$ in $r(t)$, since $l(t)$ is independent of $m(t)$, we may consider the functions $r(t)$ and $m(t)$ as independent.

The case $H(p)=\eta / p$ is therefore appropriate to represent the two required, physically different, memory mechanisms acting in the anelastic media.

The memory functions $m(t)$ and $r(t)$ must satisfy the requirements set by the laboratory data or those observed in nature which, in general, concern the dissipation of energy, that is the $Q$, and the dispersion caused by the frequency dependent velocity.

But we must also take into account the phenomenon of dispersion forecasted for monochromatic electromagnetic waves travelling in homogeneous dielectric solids or plasmas, and also for waves travelling in anelastic media; the dispersion of a monochromatic wave is caused by the new constitutive equations and appears when they contain the imaginary frequency elevated to a rational power. It consists in a splitting of the wave into a set of waves with the same frequency but slightly different wavelength (e.g., Caputo, 1993a).

From an experimental point of view, the independence of $r(t)$ and $m(t)$ is of great help for their determination and one may envisage the physically different laboratory methods to ob- 
tain the functions $r(t)$ and $m(t)$. Concerning the Earth, from an observational point of view, one may theoretically obtain the two functions using separately the data on the dissipation of energy and dispersion of the free torsional modes and of the purely radial modes.

The separation of the dilatation effect from the shear effect, due to the presence of the memory functions in (3.1), allows the indexes of refraction of the $P$ and $S$ waves to be estimated.

From (3.2) we find that

$$
\begin{gathered}
\{\lambda+2 \mu\} \rightarrow[(3 L+2 M) \mu+ \\
+3(L+2 M) p H] / 3 H(\mu+p H)
\end{gathered}
$$

and we may obtain from (3.5b) the index of refraction of the $P$ waves in the frequency domain

$$
\begin{aligned}
n_{p}(p)= & {[3 H(\mu+p H)(\lambda+2 \mu) /\{(3 L+2 M) \mu+} \\
& +3(L+2 M) p H\}]^{1 / 2}
\end{aligned}
$$

while for the $S$ waves we find from the second of (3.2)

$$
n_{s}(p)=[\mu(\mu+p H) / p M]^{1 / 2} \text {. }
$$

In (3.6a) and (3.6b) it is assumed unity density.

Formula (3.6b) is also useful in the computations of the free torsional modes of homogeneous spheres or of spherical shells and of homogeneous layers limited by planes. For Raleigh surface waves (spheroidal modes of homogeneous spheres or spherical shells) the expression of the index of reflaction is more complicated but it may be obtained from (3.5a).

Other forms of representing the dissipation of energy and the dispersion in anelastic media have been used (e.g., Caputo, 1986, 1993b); the form (3.1) is one of the most comprehensive and, with $H(p)=\eta / p$, it may be reduced to any of the other forms used.

Concerning the dielectric media it is generally accepted that the dielectric parameter, relating the applied electric field vector $E$ to the induction vector $D$, is a tensor of the second order.
The experimental data suggested (Cole and Cole, 1941) that in one dimension and in the LT domain the dielectric parameter is represented by

$$
\left(\alpha+\gamma p^{z}\right) d=\left(\sigma+\eta p^{z}\right) e
$$

where $p=i \omega, d$ and $e$ are the LT of $D$ and $E$ respectively and $z$ is a positive real number generally less than one.

Equation (3.6a) is the LT of the corresponding time domain relation (Caputo and Mainardi, 1971a)

$$
\left(\alpha+\gamma \partial^{z} / \partial t^{z}\right) D=\left(\sigma+\varepsilon \partial^{z} / \partial t^{z}\right) E .
$$

A general expression of stress-strain relations has been introduced by Caputo (1986, 1993b) allowing it to be reduced appropriately specializing the parameters, to the old expressions of Kelvin-Voigt or Maxwell, whatever this may be useful for besides giving the feeling that the new form contains as a special case the old unsuccessful forms.

Caputo's (1966, 1986, 1993b) expressions for the memory mechanisms are formulated in terms of fractional order derivatives; generalizing these expressions in order to allow more flexibility in adapting the formulae to the experimental data we obtain

$$
\begin{gathered}
h^{*} \tau_{i j}^{\prime}+\mu\left(\tau_{i j}-\delta_{i j} \tau_{r r} / 3\right)= \\
=\lambda \delta_{i j} h^{*} \varepsilon_{r r}^{\prime}+2 \mu h^{*} \varepsilon_{i j}^{\prime}+n_{1}^{*} \delta_{i j} \lambda \varepsilon_{r r}+2 n_{1}^{*} \mu \varepsilon_{i j}
\end{gathered}
$$

which gives in the LT domain

$$
\begin{gathered}
H p T_{i j}+\mu\left(T_{i j}-T_{r r} / 3\right)= \\
=\left(\lambda \delta_{i j} E_{r r}+2 \mu E_{i j}\right) p H+N_{1}\left(\lambda \delta_{i j} E_{r r}+2 \mu E_{i j}\right)
\end{gathered}
$$

which in turn allow the equilibrium equation in the LT domain to be obtained with the substitution

$$
\begin{aligned}
\{\lambda\} \rightarrow & {\left[\mu(\lambda+2 \mu / 3)\left(1+N_{1} / p H\right)+\right.} \\
& \left.+\lambda\left(p H+N_{1}\right)\right] /(\mu+p H) \\
\{\mu\} \rightarrow & \left(\mu p H+N_{1}\right) /(\mu+p H) .
\end{aligned}
$$


With (3.11) we obtain also the expressions

$$
\begin{gathered}
\{\lambda+2 \mu\} \rightarrow\left[\mu(\lambda+2 \mu / 3)\left(1+N_{1} / p H\right)+\right. \\
\left.+(\lambda+2 \mu)\left(p H+N_{1}\right)\right] /(\mu+p H) \\
\{3 \lambda+2 \mu\} \rightarrow(3 \lambda+2 \mu)\left(p H+N_{1}\right) / p H .
\end{gathered}
$$

Formulae (3.11) and (3.12) readily give the index of refraction of the $P$ and $S$ waves.

In (3.12) it is also seen that for $N_{1}=0$, the expression of the incomprehensibility becomes independent of time and there is no memory in the quasistatic dilatation.

These formulae appear in different forms in the article of Caputo (1993b) where derivatives of fractional order are used for $h(t)$ and $n_{1}(t)$ (the latter with a slightly different expression) and where the second order derivatives of the strain are also considered.

The experimental data available do not yet allow the three dimensional properties of the dielectric tensor to be formulated; moreover, in the field of studies of dielectric media, almost all problems considered are reduced to the onedimensional form; the same is true also in the applications to prospecting problems. In these cases the relation between $D$ ad $E$ is of the type

$B(t)^{*} D=F(t)^{*} E$ or $d=[f(p) / b(p)] e$

where $d$ and $e$ are the LT of $D$ and $E$ respectively. Equation (3.13) may be readily specialized in the simpler form (3.7) which is widely used for field or laboratory research in the frequency domain, although recently (Pelton et al., 1983, Patella et al., 1987; Caputo 1993a, 1996b; Caputo and Plastino, 1996) the use of the time domain form has become more frequent.

The index of refraction associated to (3.13) is obviously

$$
n=[f(p) / b(p)]^{1 / 2} / c
$$

where $c$ is the velocity of light and it is assumed that magnetic permeability be unity.

In the study of one-dimensional problems of anelastic media the stress-strain relation is also expressed in the form (3.9) or (3.7).

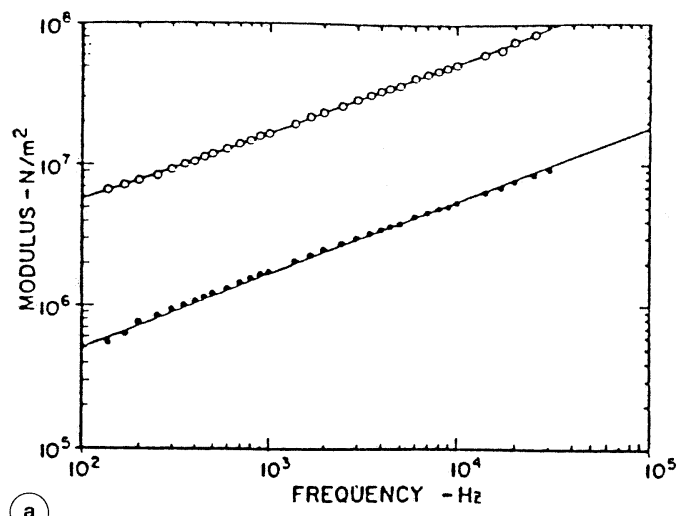

(a)
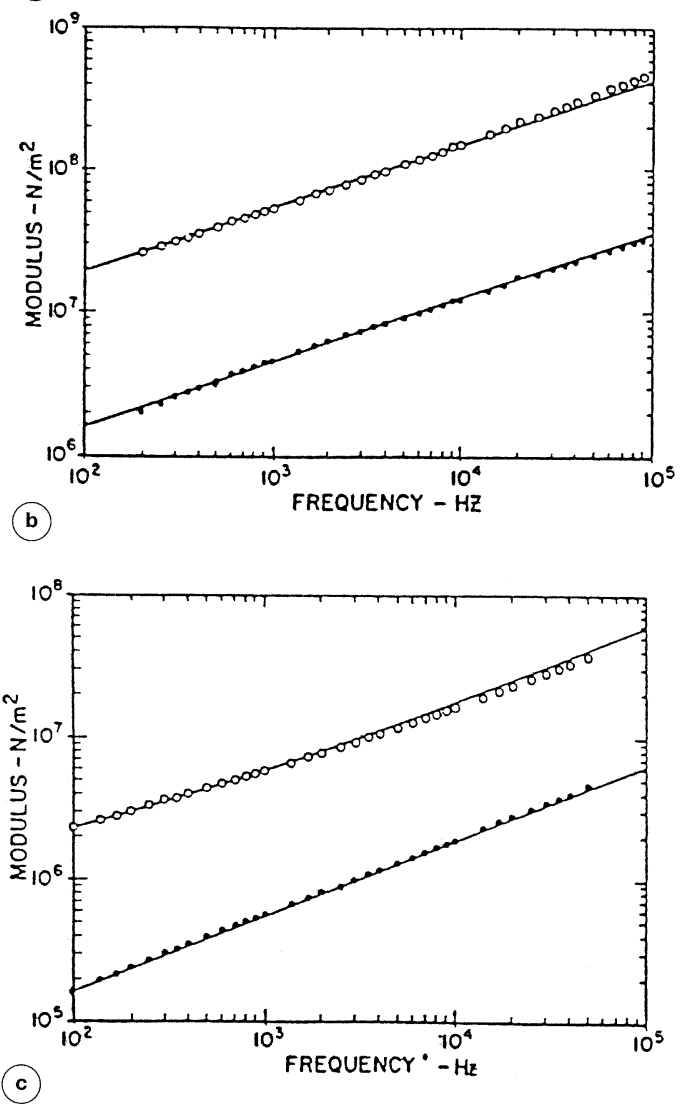

Fig. 1a-c. The mechanical viscoelastic properties of: a) butyl $\mathrm{B} 252$ at $10^{\circ} \mathrm{C}$; storage modules, dissipation modules (after Bagley and Torvik, 1983a); b) butyl 70821 at $10^{\circ} \mathrm{C}$ (after Bagley and Torvik, 1983a); c) polybutadine at $10^{\circ} \mathrm{C}$ (after Bagley and Torvik, 1983a). 
Many problems of anelastic and dielectric media are solved by introducing the index of reflaction; it is therefore obvious that these problems of anelastic and dielectric media, when in the latter the time variation of the current density of free charges is nil, have the same mathematical equations. Depending on the boundary conditions, the solutions are therefore expressed in the same mathematical form and have the same properties.

\section{The constitutive equations with deriva- tives of rational order (or the multivalued index of refraction)}

For a long time mathematics and physics have focussed on the modelling of the dissipation and dispersion of waves, propagation of energy and perturbations in solid anelastic media, plasmas, fluid and solid dielectrics.

Heaviside (1889), with his symbolic calculus, studied the propagation of electromagnetic energy, later Cisotti (1911), introduced memory mechanisms to represent dispersion and dissipation, followed by Graffi (1936) and by Cole and Cole (1941) who studied induced polarization assuming a frequency dependent complex dielectric parameter. In the studies of the dissipation of elastic energy the work of Bagley and Torvik (1983a,b) is fundamental as shown in figs. 1a-e.

The use of derivatives of real order $z$ which were first systematically studied by Liouville (1832), is now becoming more popular in applied physics especially in the formulation of the constitutive equations of dispersive media and description of diffusive phenomena. In fact from problems of dissipation of energy in anelastic media (Caputo 1969; Caputo and Mainardi, 1971a, Bagley and Torvik, 1983a,b), the use of fractional derivatives is now extended to the study of the rheology of the Earth (Körnig and Müller, 1989), to diffusion problems (Mainardi, 1994; Caputo 1996a), to electric energy storage (Jacquelin, 1991), to geoelectric prospecting (Pelton et al., 1983; Caputo, 1996b; Caputo and Plastino, 1996), to the dispersion and attenuation of waves in plasmas and fluid media (Caputo, 1995a,b).
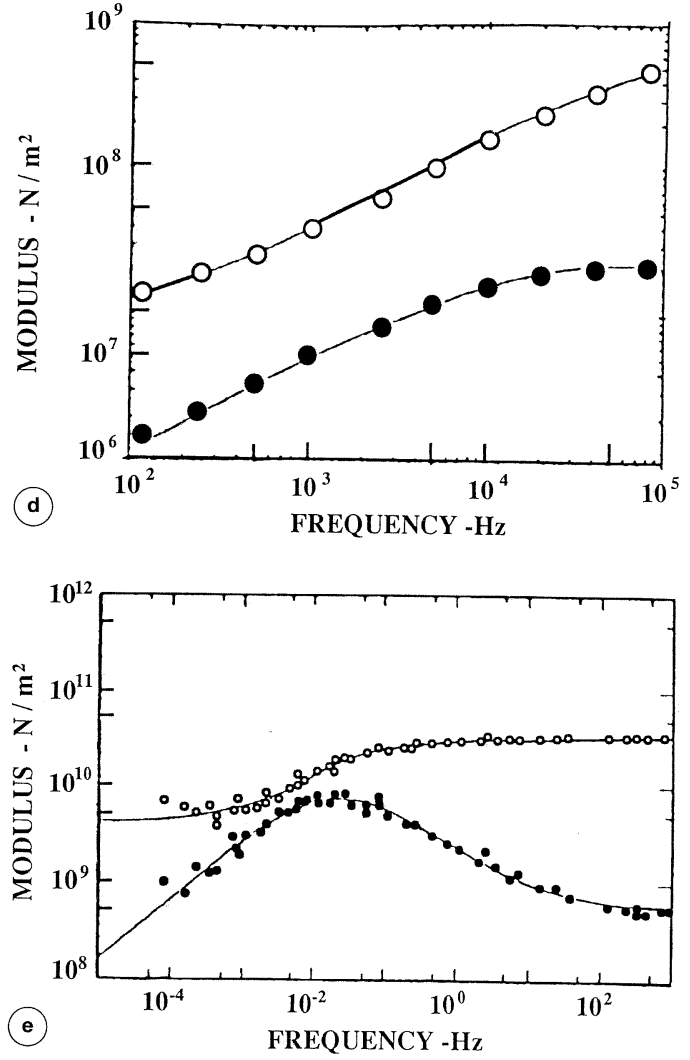

Fig. 1d,e. The mechanical viscoelastic properties of: d) Nitrile Rubber 1479 at $10^{\circ} \mathrm{C}$; storage modules, dissipation modules (after Bagley and Torvik, 1986); e) Corning 10 at $10^{\circ} \mathrm{C}$ (after Bagley and Torvik, 1983a).

It is often, but not always (Caputo, 1986), assumed that the memory functions described in section 3 are represented by derivatives of fractional order in the constitutive relation of the medium. A most general form to introduce these derivatives in the constitutive relations (3.1) of section 3 is to assume

$$
\begin{aligned}
& l(t)=\eta_{1} \lambda t^{-z_{1}} / \Gamma\left(1-z_{1}\right) \\
& h(t)=\eta_{2} t^{-z_{2}} / \Gamma\left(1-z_{2}\right) \\
& m(t)=\eta_{3} \mu t^{-z_{3}} / \Gamma\left(1-z_{3}\right) \\
& L(p)=\eta_{1} \lambda p^{z_{1}-1} \\
& H(p)=\eta_{2} p^{z_{2}-1} \\
& M(p)=\eta_{3} \mu p^{z_{3}-1}
\end{aligned}
$$


where $\eta_{1}, \eta_{2}$ and $\eta_{3}$ have dimensions $N s^{z_{1}} m^{-1}$, $N s^{z_{2}} m^{-1}, N s^{z_{3}} m^{-1}$ respectively.

In this case the LT of the equilibrium equations are obtained with the substitution

$$
\begin{aligned}
\{\lambda\} \rightarrow & \left(3 \lambda \mu \eta_{1} p^{z_{1}}+2 \mu^{2} \eta_{3} p^{z_{3}}+\right. \\
& \left.+3 \eta_{1} \eta_{2} \lambda p^{z_{1}+z_{2}}\right) / 3 \eta_{2} p^{z_{2}}\left(\mu+\eta_{2} p^{z_{2}}\right) \\
\{\mu\} \rightarrow & \eta_{3} \mu p^{z_{3}} /\left(\mu+\eta_{2} p^{z_{2}}\right)
\end{aligned}
$$

which give

$$
\begin{gathered}
\{\lambda+2 \mu\} \rightarrow\left[\mu\left(3 \eta_{1} \lambda p^{z_{1}}+2 \eta_{3} \mu p^{z_{3}}\right)+\right. \\
\left.+3 \eta_{2} p^{z_{2}}\left(\lambda \eta_{1} p^{z_{1}}+2 \mu \eta_{3} p^{z_{3}}\right)\right] /\left[3 \eta_{2} p^{z_{2}}\left(\mu+\eta_{2} p^{z_{2}}\right)\right] .
\end{gathered}
$$

Assuming unity density and remembering (3.5b), the indexes of refraction of the $P$ and $S$ waves are then

$$
\begin{aligned}
n_{p}= & \left\{3 \eta_{2} p^{z_{2}}\left(\mu+\eta_{2} p^{z_{2}}\right) /\left[\left(3 \lambda \eta_{1} p^{z_{1}}+2 \mu \eta_{3} p^{z_{3}}\right) \mu+\right.\right. \\
& \left.\left.+3\left(\lambda \eta_{1} p^{z_{1}}+2 \mu \eta_{3} p^{z_{3}}\right) \eta_{2} p^{z_{2}}\right]\right\}^{1 / 2} \\
n_{s}= & \left\{\left(\mu+\eta_{2} p^{z_{2}}\right) / \mu \eta_{3} p^{z_{3}}\right\}^{1 / 2}= \\
= & {\left[\mu / \eta_{3} p^{z_{3}}+\eta_{2} / \eta_{3} p^{z_{3}-z_{2}}\right]^{1 / 2} . }
\end{aligned}
$$

From which one may obtain the velocities as the inverse of the real part of the index of refraction.

It is also clear that when we set

$$
z_{1}=z_{2}=z_{3}=z, \quad \eta_{1}=\eta_{2}=\eta_{3}=\eta
$$

in (4.2a), as was done in Caputo (1984), then

$$
\{3 \lambda+2 \mu\} \rightarrow 3 \lambda+2 \mu
$$

and there is no memory in the quasistatic dilatation; for the $P$ and $\mathrm{S}$ waves the indexes of refraction are

$$
\begin{aligned}
& n_{p}=\left\{\left[\left(1+(4 \mu /(3 \lambda+2 \mu))\left(\mu / \eta p^{z}\right)\right] /(\lambda+2 \mu)\right\}^{1 / 2}\right. \\
& n_{s}=\left\{\left(1+\mu / \eta p^{z}\right) / \mu\right\}^{1 / 2}
\end{aligned}
$$

If in (4.6) and (4.7) $z=r / m$ with $r$ and $m$ positive, integer and prime, then the $z$-th power of $p$ has $m$ values. However there is the condition $1 / Q>0$ which implies $\operatorname{Re}(n)>0$ since we take $\operatorname{Im}(n)>0$; in general the two requirements give about $m / 4$ physically acceptable velocity fields.

The $1 / Q$ of the $P$ and $S$ waves are readily obtained from (4.6). When $\eta p^{z} / \mu \ll 1$, setting $p=i \omega$ it is

$$
\begin{gathered}
1 / Q_{p}=4\left(\mu / \eta \omega^{z}\right) \sin ((0.5+2 k) \pi z / 2) \\
1 / Q_{s}=\left(\mu / \eta \omega^{z}\right) \sin ((0.5+2 k) \pi z / 2) \\
k=0,1,2, \ldots, m-1 .
\end{gathered}
$$

Let us now go to the second model of stress-strain relations presented in eq. (3.9) of section 3. Assuming fractional order derivatives for $h(t)$ and $n_{1}(t)$ in formulae (3.11) and (3.12) of section 3 , that is

$$
\begin{aligned}
& h(t)=\eta t^{-z} / \Gamma(1-z), \quad H=\eta p^{z-1} \\
& n_{1}(t)=\eta_{1} t^{-z_{1}} / \Gamma\left(1-z_{1}\right), \quad N_{1}=\eta_{1} p^{z_{1}-1}
\end{aligned}
$$

we obtain

$$
\begin{aligned}
& \{\lambda\} \rightarrow\left[\mu(\lambda+2 \mu / 3)\left(1+\eta_{1} p^{z_{1}-1} / \eta p^{z}\right)+\right. \\
& \left.+\lambda\left(\eta p^{z}+\eta_{1} p^{z_{1}-1}\right)\right] /\left(\mu+\eta p^{z}\right) \\
& \{\mu\} \rightarrow \mu\left(\eta p^{z}+\eta_{1} p^{z_{1}-1}\right) /\left(\eta p^{z}+\mu\right) \\
& \{\lambda+2 \mu\} \rightarrow\left[\mu(\lambda+2 \mu / 3)\left(1+\eta_{1} p^{z_{1}-z-1} / \eta\right)+\right. \\
& \left.+(\lambda+2 \mu)\left(\eta p^{z}+\eta_{1} p^{z_{1}-1}\right)\right] /\left(\mu+\eta p^{z}\right) \\
& \{3 \lambda+2 \mu\} \rightarrow\left[\mu(3 \lambda+2 \mu)\left(1+\eta_{1} p^{z_{1}-z-1} / \eta\right)+\right. \\
& \left.+(3 \lambda+2 \mu)\left(\eta p^{z}+\eta_{1} p^{z_{1}-1}\right)\right] /\left(\mu+\eta p^{z}\right)
\end{aligned}
$$

which reproduce Hooke's law when setting $\eta_{1}=0$ and then taking the limit for $\eta \rightarrow \infty$.

From (4.12) and (4.13) the indexes of refraction of the $P$ and $\mathrm{S}$ wave are readily obtained. The case when $\eta_{1}=0$ is particularly simple, it was successfully used by Körnig and Müller (1989) to represent the rheology of the 
mantle of the Earth; its indexes of refraction coincide with (4.6). It is to be noted that, when $\eta_{1}=0,(4.14)$ give

$$
\{3 \lambda+2 \mu\} \rightarrow(3 \lambda+2 \mu)
$$

and there is no Thompson effect in quasi static experiments; the same would be true for $(4.2 \mathrm{a})$ when (4.4) applies; we note also that, when (4.4) applies, (4.2a) coincide with (4.14) when $\eta_{1}=0$.

The presence of the rational powers of $p$ in (4.6) through (4.14) implies that both $n_{p}$ and $n_{s}$ are multivalued functions and, therefore, in the medium there is more than one velocity field for the $P$ and the $\mathrm{S}$ waves.

From a monochromatic source, in a given direction and with a given frequency, is generated a set of waves with the same frequency but different wavelength and velocity. Not only the waves have different wavelength and velocity but they have also different energy dissipation.

The number of values of $n_{p}$ and $n_{s}$ for each frequency, and therefore the number of different velocity fields for each frequency, depends on the value of $z$.

\section{The dispersion in elastic waves}

When $z$ is determined experimentally, in general, it is a rational number; but if, for some reason, one must assume that it is an irrational or transcendental number, then the physically acceptable velocity fields are an infinite set.

In all cases the numerical values of each physically acceptable index of refraction, for a given frequency, are in a limited range.

This implies that a wave, originated with a given frequency at the source, entering the medium in a normal direction to the boundary, is split into a set of waves with the same frequency but with slightly different wavelength. The different wavelength is the cause of the dispersion. This is seen considering the analytic expression of the set of waves associated to the physically acceptable velocity fields given by the values of the rational power of $p$ which are, in number, about equal to the nearest integer to $m / 4$, and expressed by

$$
\begin{gathered}
p_{k}=|p|\{\cos ((0.5+2 k) \pi z / 2)+\sin ((0.5+ \\
+2 k) \pi z / 2)\} \\
k=0,1, \ldots \ldots, m-1
\end{gathered}
$$

set in the analytic expression of the index of refraction. by

The set of waves is analytically described

$\sum_{k} A_{k}(x, f) \sin \left[2 \pi f\left(x \operatorname{Re}\left(n_{k}\right)-t\right)+\varphi_{k}\right]$

where the sum is extended to all the values of $k$ corresponding to the physically acceptable indexes of refraction $n_{k}$ and where $A_{k}(x, f)$ incorporates the decay of the amplitude of the waves along the path, $f$ is the frequency, $x$ is the length of the ray from the source to the point where we assume that the $k$-th wave has the same phase $\varphi_{k}$ as at the initial time at the source.

A wave of the set, with amplitude $A_{k}$ at the source, at the distance $x$ will have amplitude

$$
A_{k}(x, f)=A_{k} \exp \left(-\pi f x \operatorname{Re} n_{k} / Q\right)
$$

where the $1 / Q$ of the wave appears.

Equation (5.2) may then be written

$$
\sum_{k} A_{k} \exp \left(-f x \pi \operatorname{Re} n_{k} / Q\right) \sin \left[2 \pi f\left(x \operatorname{Re}\left(n_{k}\right)-t\right)+\varphi_{k}\right] .
$$

In order to discuss the amplitude of the set of waves at the distance $x$ let us assume in (5.1) and therefore in (5.4)

$$
\vartheta_{k}=(0.5+2 k) r \pi / m
$$

with

$$
\begin{gathered}
\mu / 2 \eta \omega^{z} \ll 1 \\
1 / Q=\left[\left(\mu / \eta \omega^{z}\right) \sin \vartheta_{k}\right]>0 .
\end{gathered}
$$

If the number of physically acceptable values of $k$ is sufficiently large, then we may reasonably assume that the values of

$$
\vartheta_{k}-2 q_{k} \pi
$$


with $q_{k}>0$ integer and with the appropriate value of $q_{k}>0$ for each $k$, be uniformly distributed in the range $0, \infty$. As a consequence we may write (5.2)

$$
\begin{aligned}
& \sum_{k}\left[\operatorname { e x p } \left\{\left(-\pi f x / \mu^{1 / 2}\right)(1+\right.\right. \\
& \left.\left.\left.+\left(\mu / 2 \eta \omega^{z}\right) \cos \vartheta_{k}\right)\left(\mu / 2 \eta \omega^{z}\right) \sin \vartheta_{k}\right\}\right] \\
& \sin \left(2 \pi f\left(\left(x / \mu^{1 / 2}\right)\left(1+\left(\mu 2 \eta \omega^{z}\right) \cos \vartheta_{k}\right)-t\right)+\varphi_{k}\right)= \\
& =\sin \left(2 \pi f\left(x / \mu^{1 / 2}-t\right)\right) \sum_{k} A_{k}(x, f) \\
& \cos \left(\left(\pi f x \mu^{1 / 2} / \eta \omega^{z}\right) \cos \vartheta_{k}+\varphi_{k}\right)+ \\
& +\cos \left(2 \pi f\left(x / \mu^{1 / 2}-t\right)\right) \sum_{k} A_{k}(x, f) \\
& \sin \left(\left(\pi f x \mu^{1 / 2} / \eta \omega^{z}\right) \cos \vartheta_{k}+\varphi_{k}\right)= \\
& =V \sin \left(2 \pi f\left(x / \mu^{1 / 2}-t\right)\right)+U \cos \left(2 \pi f\left(x / \mu^{1 / 2}-t\right)\right)= \\
& =\left(U^{2}+V^{2}\right)^{1 / 2} \sin \left[2 \pi f\left(x / \mu^{1 / 2}-t\right)+\right. \\
& \left.+\sin ^{-1}\left(U /\left(U^{2}+V^{2}\right)^{1 / 2}\right)\right] \\
& U=\sum_{k} A_{k}(x, f) \sin \left[\left(\omega^{1-z} x \mu^{1 / 2} / 2 \eta\right) \cos \vartheta_{k}+\varphi_{k}\right] \\
& V=\sum_{k} A_{k}(x, f) \cos \left[\left(\omega^{1-z} x \mu^{1 / 2} / 2 \eta\right) \cos \vartheta_{k}+\varphi_{k}\right] \\
& A_{k}(x, f)=A_{k} \exp \left[\left(-\omega^{1-z} x \mu^{1 / 2} / 2 \eta\right) \sin \vartheta_{k}\right]
\end{aligned}
$$

where again the sum is extended to all the values of $k$ corresponding to the physically acceptable values of the index of refraction, which imply that $\sin ((0.5+2 k) \pi r / m)>0$ and that $\cos ((0.5+2 k) \pi r / m)>0$, in the expression of the amplitude $A_{k}(x, f)$; it is also assumed that $\mu / 2 \eta \omega^{z} \ll 1$ and therefore that $\left(\mu / 2 \eta \omega^{z}\right)^{2}$ be negligible.

In the expressions (5.8) it is confirmed that all the waves of the set have the same frequency and, with the assumption that the waves at the origin have given phases, the formula gives, at the distance $x$, the phases of the single waves and the amplitude of their sum.

The group velocity is

$$
\mu^{1 / 2}\left\{1-\left(\mu^{1 / 2} / \omega\right)[V(d U / d x)-U(d V / d x)] /\left(U^{2}+V^{2}\right)\right\}
$$

which shows that, if $U=0$ (or $V=0$ ), the group velocity coincides with the average phase velocity $\mu^{1 / 2}$ of the set of waves.

There are cases, as in the atmosphere of the Earth, when the $1 / Q$ of all the waves of the set is almost negligible; if we assume that $\varphi_{k}=0$ and that there is equipartition of energy among them, then

$$
A_{k}(x, f)=A(x, f)=A
$$

and the sum (5.2) is reduced to the simpler expression

$$
\begin{aligned}
& \left(U_{1}^{2}+V_{1}^{2}\right)^{1 / 2} \sin \left[2 \pi f\left(x / \mu^{1 / 2}-t\right)+\right. \\
& \left.\quad+\sin ^{-1}\left(U_{1} /\left(V_{1}^{2}+U_{1}^{2}\right)^{1 / 2}\right)\right] \\
& U_{1}=A \sum_{k} \sin \left[\left(\omega^{1-z} x \mu^{1 / 2} / 2 \eta\right) \cos \vartheta_{k}\right] \\
& V_{1}=A \sum_{k} \cos \left[\left(\omega^{1-z} x \mu^{1 / 2} / 2 \eta\right) \cos \vartheta_{k}\right] \\
& B_{1}(x, f)=\left(U_{1}^{2}+V_{1}^{2}\right)^{1 / 2}
\end{aligned}
$$

where $B_{1}$ is the amplitude of the set of waves.

Because of (5.10) we may write

$[V(d U / d x)-U(d V / d x)]=$

$$
\begin{aligned}
& =A \sum_{k} \cos \vartheta_{k} \sum_{j}\left(\cos \left(\left(\omega^{1-z} x \mu^{1 / 2} / 2 \eta\right) \cos \vartheta_{k}\right)\right. \\
& \cos \left(\left(\omega^{1-z} x \mu^{1 / 2} / 2 \eta\right) \cos \vartheta_{j}\right)+ \\
& +\sin \left(\left(\omega^{1-z} x \mu^{1 / 2} / 2 \eta\right) \cos \vartheta_{k}\right) \\
& \left.\sin \left(\left(\omega^{1-z} x \mu^{1 / 2} / 2 \eta\right) \cos \vartheta_{j}\right)\right)+A \sum_{k} \cos \vartheta_{k}
\end{aligned}
$$

where we have extended the sum to all the couples $k$ and $j$, which give physically acceptable velocity fields, and the last term of the equation results from the terms of the sum with $k=j$.

$$
A \sum_{k} \cos \vartheta_{k}
$$

$\left[1+\sum_{j} \cos \left\{\left(x \omega^{1-z} \mu^{1 / 2} / 2 \eta\right)\left(\cos \vartheta_{k}-\cos \vartheta_{j}\right)\right\}\right]$.

If the number of values of $k$, which give physically acceptable velocity fields is sufficiently large, then (5.13) is nil and the phase and 
group velocities are equal. The same result is verified considering that when the number of values of $k$ which give the physically acceptable velocity field is sufficiently large, then (5.12) may be written

$$
\begin{aligned}
& U_{1}=A(m / \pi) \int_{0}^{\pi} \sin \left(\left(\omega^{1-z} \mu^{1 / 2} x / 2 \eta\right) \cos \vartheta\right) d \vartheta \cong 0 \\
& V_{1}=2 A(m / \pi) \int_{0}^{\pi / 2} \cos \left(\left(\omega^{1-z} \mu^{1 / 2} x / 2 \eta\right) \cos \vartheta\right) d \vartheta
\end{aligned}
$$

which imply that (5.2) is

$$
V_{1} \sin \left(2 f\left(x / \mu^{1 / 2}-t\right)\right)
$$

The amplitude $V_{1}$ (as well as $V$ ) is a quasi cosinusoidal function of $x$ with slowly varying wavelength. The first four zeros of the amplitude $V_{1}$ are at the values of $x$ solution of the equation

$$
\begin{aligned}
& Z=\left(\omega^{1-z} \mu^{1 / 2} / 2 \eta\right) x \\
& Z=2.405,5.520,8.654,11.790 .
\end{aligned}
$$

Note that the values of $Z$ in (5.16) are separated by something less than $\pi$; the following ones are separated by a quantity which is increasingly closer to $\pi$. In fact if the amplitude $V_{1}$, is nil for a value of $Z$ larger than those given in (5.16), then it is readily verified numerically that, for not too large values of the integer $q$, which would cause instability in the numerical integration because of the limited number of digits in $\pi$, one finds

$$
\int_{0}^{\pi / 2} \cos ((Z+q \pi) \cos \vartheta) d \vartheta=0
$$

Increasing $x$, the amplitude of the sum of the set of waves varies periodically, its wavelength is

$$
\Lambda=2 \eta \omega^{z} / f \mu^{1 / 2}
$$

which decreases with increasing frequency and phase velocity, while the average wavelength of the waves of the set is $\mu^{1 / 2} / f$ and their ratio is simply $\mu / 2 \eta \omega^{z}$ which is relatively large since we have assumed $2 \eta \omega^{z} / \mu^{1 / 2} \ll 1$.
The beats and the zeros of the amplitude of the sum of the waves of the set with the same frequency and direction at the source are generated by interference. When $\varphi_{k}$ depend on time and/or there is variation in the partition of energy among the waves of the set, then the zeros of $V_{1}$, and its maxima as well, move along the $x$ axis; the same is true for the energy and in each point there is an average energy present which can, depending on $\varphi_{k}$, vary between the maximum and zero.

The phases of the different waves of the set with the same frequency, direction and phase at the source will also arrive at the same distance at slightly different times.

It is seen that, due to the different decay of the waves of the set, after some distance, some waves may have negligible amplitude and give no contribution to the average wavelength of the set which will necessarily change with the distance from the source. The disappearance of the waves along the path will also change the average velocity and the amplitude of the set of waves.

\section{The dispersion in dielectric media and plasmas}

The discussion of the dielectric media with frequency dependent index of refraction follows the same pattern as that of anelastic media (Caputo, 1994); in the case of dielectric media however the dielectric parameter is often used as a scalar with the following general form

$$
\left(\sigma+\eta(i \omega)^{z}\right) /\left(\alpha+\gamma(i \omega)^{z}\right)
$$

where it is assumed in practice (Cole and Cole, 1941)

$$
\alpha=1, \quad \sigma / \alpha=\varepsilon_{0}, \quad \eta / \gamma=\varepsilon_{\infty}, \quad \gamma / \alpha=t_{1}^{z} .
$$

The index of refraction is then readily found

$$
\begin{aligned}
& n=n_{r}-i n_{i}=\varepsilon_{0}^{1 / 2}\left\{\left[1+(1+a) o c_{k}+a o^{2}+\right.\right. \\
& \left.\left.+i o(a-1) s_{k}\right] /\left[1+2 o c+o^{2}\right]\right\}^{1 / 2}
\end{aligned}
$$


where

$o=\left(t_{1} \omega\right)^{z}, \quad a=\varepsilon_{\infty} / \varepsilon_{0}$,

$c_{k}=\cos \vartheta_{k}, s_{k}=\sin \vartheta_{k}, \quad k=0,1,2, \ldots, m-1$

From (6.3) and (6.4) one obtains

$$
\begin{aligned}
n_{r}= & q \cos \psi_{k}, \quad n_{i}=q \sin \psi_{k} \\
q= & \varepsilon_{0}^{1 / 2}\left[\left(1+(1+a) o c_{k}+a o^{2}\right)^{2}+\right. \\
& \left.+o^{2}(a-1)^{2} s_{k}^{2}\right]^{1 / 4} /\left[1+2 o c_{k}+o^{2}\right]^{1 / 2} \\
\psi_{k} & =0.5 \operatorname{tgh}^{-1}\left[o(a-1) s_{k} /\left(1+(a+1) o c_{k}+a o^{2}\right)\right]
\end{aligned}
$$

where $t_{1}$ is the relaxation time. Relations (6.5) define the phase velocities and the $Q$ for every $k$.

When the index of refraction of the dielectric medium is known, the discussion on the phenomena caused by its multivalued property is the same as that presented for anelastic media. The case of propagation of waves in water has been numerically discussed with some details in a paper by Caputo (1994).

In the case of water the index of refraction at $5^{\circ} \mathrm{C}$ is (Hasted, 1973)

$$
\begin{aligned}
& {\left[\varepsilon_{0}\left(1+\left(\varepsilon_{\infty} / \varepsilon_{0}\right)\left(i t_{1} \omega\right)^{z}\right) /\left(1+\left(i t_{1} \omega\right)^{z}\right)\right]^{1 / 2}=} \\
& \quad=\left[9.2608\left(1+0.0499\left(9.58210^{-11} \text { if }\right)^{0.965}\right) /\right. \\
& \left.\quad /\left(1+\left(9.58210^{-11} \text { if }\right)^{0.965}\right)\right]^{1 / 2}
\end{aligned}
$$

which we may write for frequencies smaller than $100 \mathrm{MHz}$

$$
\begin{aligned}
\varepsilon_{0}^{1 / 2}\left(1-\left(i t_{1} \omega\right)^{z} / 2\right)^{1 / 2}= \\
=9.2608\left(1-\left(9.58210^{-11} f\right)^{0.965}\right. \\
\left.\quad\left(\cos \vartheta_{k}+i \sin \vartheta_{k}\right) / 2\right) \\
m=200, \quad r=0,1,2, \ldots, 193 .
\end{aligned}
$$

It is to be noted that here the $1 / Q$ is

$$
1 / Q=\left(t_{1} \omega\right)^{z} \sin \vartheta_{k}
$$

that is, the $1 / Q$ is directly proportional to the $z$ power of the frequency while in the case previ- ously considered, of the $S$ waves in solids, the $1 / Q$ was inversely proportional to the $z$ power of the frequency.

The zeros of the first few wavelengths of the amplitude $V_{1}$ of the sum of waves with the same frequency and direction at the source are at the value of $x$ given by the equation

$$
\begin{gathered}
L=\pi f x\left(t_{1} 2 \pi f\right)^{z} / c=x f^{1+z} \pi\left(2 \pi t_{1}\right)^{z} / c= \\
=2.225 \times 10^{-19} f^{1.965}
\end{gathered}
$$

where $c$ is the velocity of light and the values of $L$ for the first four zeros are given in (5.16) of section 5 .

In fig. 2a we see the amplitude $V_{1}$ of each of the three sets of waves with frequency $10 \mathrm{MHz}, 50 \mathrm{MHz}$ and $100 \mathrm{MHz}$ travelling in water in the case when the decay of each wave neglected (dashed line) and in the case when it is taken into account (solid line) (Caputo, 1995b). In fig. 2a one may note also that there is only a scale change in the abscissa between the three different frequencies considered and that the beats and the decay of the maxima of $V_{1}$ along the $x$ axis depend only on the parameter $f^{1+z} \pi\left(2 \pi t_{1}\right)^{z} / c$.

One may note in fig. $2 \mathrm{a}$ that the difference between the two cases, with dissipation and without dissipation, is significant; however in both cases there are beats and, in the case when the dissipation is neglected, there is an apparent decay due to the dispersion caused by the different wavelength of the waves of the set which may be considered a phenomenon of self dispersion.

When at the source the phases $\varphi_{k}$ and/or the partition of energy among the waves of the set depend on time, then there is a tunnel effect since at a generic point along $x$ there may be more or less energy depending on the distribution of the $\varphi_{k}$ and of the energy at the source.

The different velocities of the waves of a set with the same frequency and direction at the source, will also cause ambiguities when the waves are used for distance measurement in media with non negligible dissipation. It is found that in water at the temperature of $5^{\circ} \mathrm{C}$, at $1 \mathrm{~km}$ distance from the source and at 


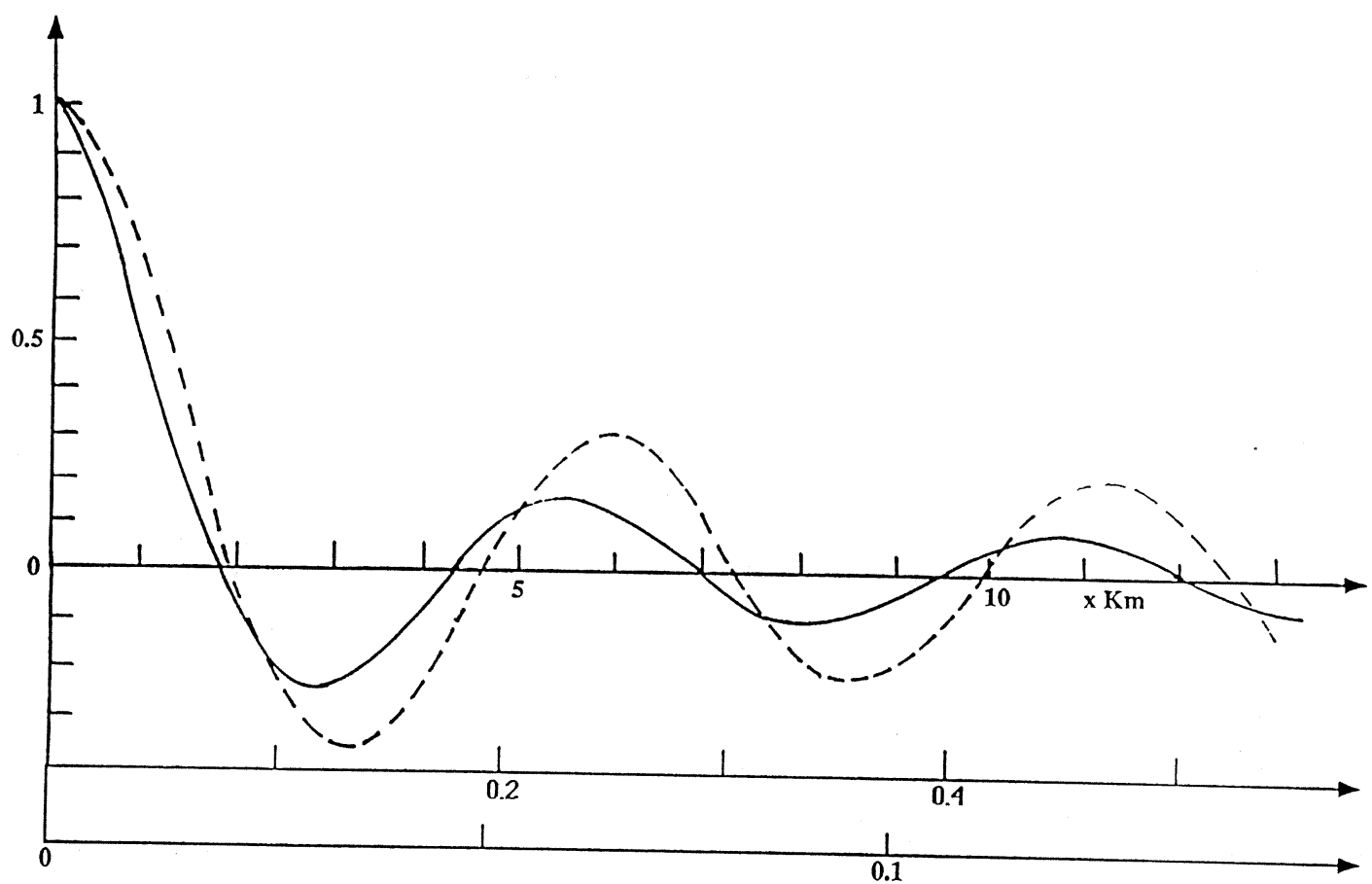

Fig. 2a. Amplitude of the set of waves with frequency $f=10 \mathrm{MHz}$ in water at $5^{\circ} \mathrm{C}\left(z=0.965, t_{1}=9.582\right.$ $10^{-11}, \varepsilon_{0}=85.7633, \varepsilon_{\infty} 4.28$ (Hasted, 1973)) as a function of the distance travelled from the source given in the abscissa. The ordinate is percent of the total amplitude at the source where the single waves are assumed to have the same amplitude and phase. The solid line is the amplitude of the real set of decaying waves. The dashed line is the theoretical amplitude of the set of waves without the effect of their decay; note the apparent space decay of this amplitude due to the different phases of the single waves of the set along the path caused by their different wavelength. The top scale of the abscissa is for $f=10 \mathrm{MHz}$, the intermediate scale is for $f=50 \mathrm{MHz}$ and the bottom scale is for $f=100 \mathrm{MHz}$ : in all cases the order of fractional differentiation is
$z=0.965$.

$10 \mathrm{MHz}$ frequency, the space spreading of the waves along the ray is about $1.22 \mathrm{~m}$, while the time spreading is about 38 ns (Caputo, 1995b).

\section{The splitting of eigenvalues and the dis- persion in media whose constitutive equa- tions contain irrational order derivatives}

\subsection{Introduction}

In most problems involving the use of derivatives of real order $z$ it is irrelevant that $z$ be a rational or an irrational number.
It has been shown however (Caputo, $1995 \mathrm{c})$, that the differentiation of rational order

$$
z=m / r<1
$$

with $m$ and $r$ positive, integer and prime, of a single valued function gives a set valued function with $r$ values. The consequences of this phenomenon have been studied by Caputo $(1990,1995 a, b)$, but thee case when $z$ is real and not rational to my knowledge, has not yet been considered. It is the topic of the next paragraphs. 


\subsection{The problems}

When trying to fit the classic problems of elastic media with frequency independent elastic constants, or of dielectric media with frequency independent dielectric parameter, to the reality of dispersion and dissipation of energy, it is now customary to consider that, phenomenologically, the dispersion and the dissipation of energy are a consequence of the presence of memory formalism in the constitutive equation of the medium.

The most common memory mechanism used is that of differentiation with real order $z$. The limitation, set in the discussion of eigenvalue problems, to the case when $z$ is a rational number, is due to a fact that $z$ is estimated experimentally and therefore is necessarily a rational number.

In the solution of the eigenvalue problems, when $z$ is rational, each eigenvalue of the classic case without dissipation or dispersion, is split into a set of $r$ eigenvalues (Caputo, 1990, 1993a, 1995a,b).

It is readily seen, following the same procedure used by Caputo (1995c) for the case when $z$ is rational, that the differentiation of real but non rational order $z$ gives a set valued function with an infinite number of values.

The discussion of the theoretical case when $z$ is real but not rational is the subject of the following paragraphs, studying the propagation of waves and the eigenvalue problems of these media.

\subsection{The propagation of the waves}

In the following we shall refer indifferently to anelastic or dielectric media considering their complex, frequency dependent, index of refraction in the form

$$
\begin{aligned}
n & =n_{r}-i n_{i}=1+(\sigma-\zeta) / 2+(\eta-\gamma)(2 \pi i f)^{z} / 2= \\
& =1+(\sigma-\zeta) / 2+\left[(\eta-\gamma)(2 \pi f)^{z} / 2\right] \\
& {[\cos (0.5+2 k) z \pi+i \sin (0.5+2 k) z \pi] }
\end{aligned}
$$

where $k$ is integer, $z$ is real but not rational, $f$ is frequency $\sigma, \zeta, \eta, \gamma$ are constants with the appropriate dimensions which appear in the complex dielectric parameter, for the dielectric case, or in the complex elastic parameter, for the anelastic case; both parameters are here represented by

$\left(1+\sigma+\eta(2 \pi f i)^{z}\right) /\left(1+\zeta+\gamma(2 \pi f i)^{z}\right)$

assuming that there is a range of $f$ where one may assume

$|\sigma| \ll 1,|\zeta| \ll 1,\left|\eta f^{z}\right| \ll 1,\left|\gamma f^{z}\right| \ll 1$.

When $z$ is not rational since $k$ may assume all integer values in the range $0, \infty$, then $n_{r}$ and $n_{i}$ are set-valued functions with an infinite number of values, in the ranges

$$
\begin{aligned}
& 1+(\sigma-\zeta) / 2-|\eta-\gamma| f^{z} / 2<n_{r}<1+(\sigma-\zeta) / 2+ \\
& +|\eta-\gamma| f^{z} / 2-|\eta-\gamma| f^{z} / 2<n_{i}<|\eta-\gamma| f^{z} / 2 .(7.5)
\end{aligned}
$$

Let us now recall that when $z$ is rational as in (7.1), the index of refraction (7.2) is a set-valued function with $r$ values, and a wave propagating from the source with frequency $f$ neglecting the $1 / Q$ of the waves of the set, is represented by (Caputo, 1995a)

$$
\Phi=\sum_{k=0}^{r-1} \sin [\omega(s(B+C \cos \Omega)-t)]
$$

where

$$
\begin{aligned}
& a=\sigma-\varsigma \\
& s=\text { distance from the source } \\
& \omega=2 \pi f \\
& B=(1+a) / c \\
& C=(2 \pi f)^{z}(\eta-\gamma) / c \\
& c=\text { reference velocity }
\end{aligned}
$$

and the sum is extended to the $r$ values of $k=0,1,2, \ldots, r-1$ if $z$ is rational as in (7.1); in this case, for any given frequency, the waves of the set (7.6) have slightly different wavelength, and their sum (7.6) has beats and is decreasing in amplitude with increasing $s$; it is 
shown in fig. $2 \mathrm{a}$ as function of the distance travelled by the wave in the case the medium be water (Caputo, 1995b).

When $z$ is real and not rational the sum (7.6) over $k$ is extended from zero to infinity and assumes all the values in the range 0,2 ; the sum is then substituted with an integral to give

$$
\int_{0}^{\pi} \sin [\omega(s(B+C \cos \Omega)-t)] d \Omega .
$$

The integration of (7.8) is limited to the range $0, \pi$ because of the condition $n_{i}>0$ in (7.2) due to the constraint that the $Q>0$.

Considering that

$$
\int_{0}^{\pi} \sin [(\cos C \cos \Omega)] d \Omega=0
$$

formula (7.7) may be simplified to

$$
\Phi=[\sin (\omega(s B-t))] \int_{0}^{\pi} \cos [(\omega C) s \cos \Omega] d \Omega .
$$

The integral appearing in (7.10) is the amplitude of the wave and is shown in fig. $2 b$ as a function of

$$
x=\omega C s .
$$

We should note that fig. $2 \mathrm{~b}$ is independent of $z$; however $z$ is implied, with $f$ in the abscissa $x$ through $C$.

Comparing figs. $2 \mathrm{a}, \mathrm{b}$ it is seen that both cases, $z$ rational and $z$ irrational the effect of the dispersion gives very similar results. The integral in (7.10) is a quasi periodic function of $s$ with decreasing amplitude.

\subsection{The eigenvalue problem}

It is seen that the eigenvalue problems of the free oscillations of infinite plates and spherical shells of anelastic and dielectric media are reduced to the solution of equations of the type (Caputo 1990, 1993a)

$$
\alpha x^{2+z}+\beta x^{2}+\zeta x^{z}+\varphi=0
$$

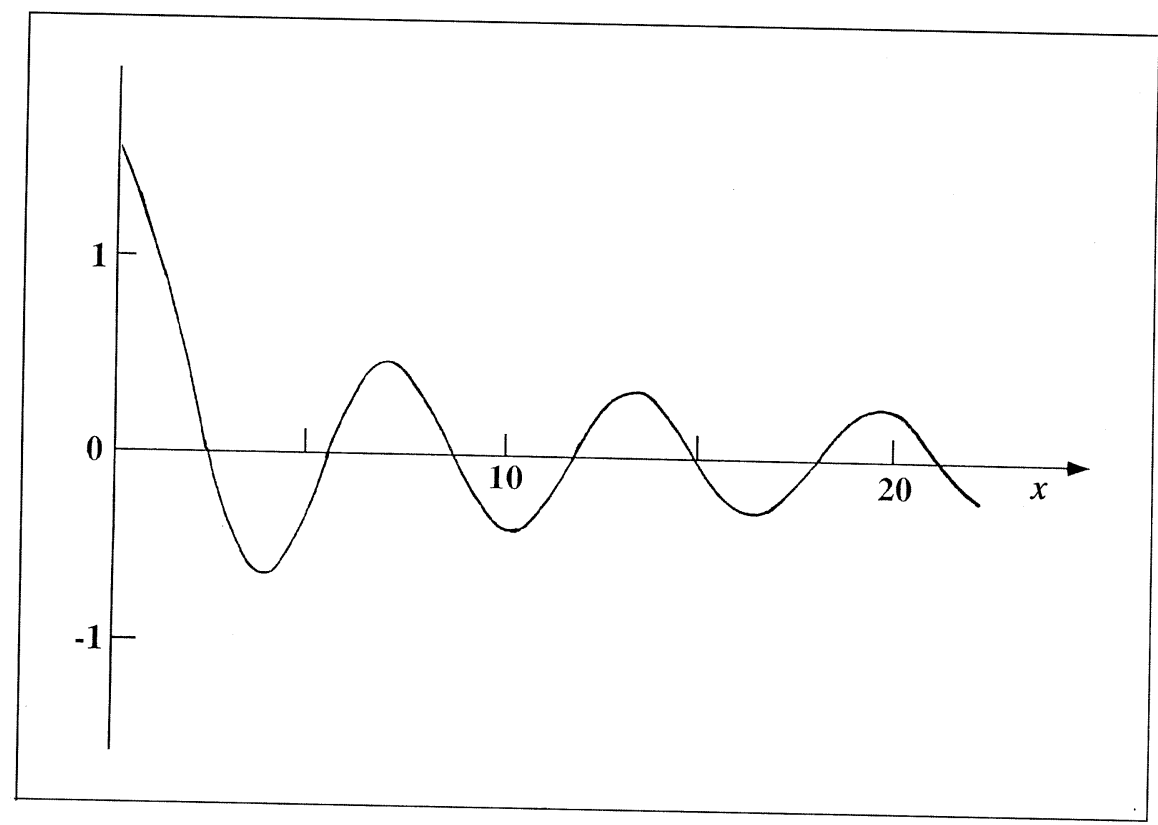

Fig. 2b. Amplitude of the set of infinite waves generated by $z$ real not rational. The abscissa is $x=C s$, defined in (7.7) and is proportional to the distance from the source. 
where

$$
x=i \omega
$$

with $\omega$ frequency and we must consider all the roots of $(i \omega)^{z}$. When $z$ is rational $(z=r / n)$

$$
x=\gamma^{n}
$$

eq. (7.12) may be written remembering (7.1), as discussed by Caputo (1993a),

$$
\alpha y^{2 n+r}+\beta y^{2 n}+\zeta y^{r}+\varphi=0
$$

which is an algebraic equation of degree $2 n+r$ and has, in general, $2 n+r$ non multiple solutions. In order to be physically acceptable, however, the solutions must satisfy the condition $\operatorname{Re}(i \omega)<0$ in order to ensure time convergence at infinite time.

When $\alpha=0, \beta \ll \varphi(7.15)$ in general has no multiple solutions, they are in the complex plane and, in the first approximation, they are on two closed curves, one in the $\operatorname{Im} y>0$ half plane and one in the $\operatorname{Im} y<0$ half plane, they have centers near $\pm(\varphi / \beta)^{1 / 2}$; both curves have $n$ poles (Caputo, 1990). When $\alpha \neq 0$ the solutions are set in three closed curves: $2 n$ solutions are in the two closed curves centered near $\pm(\varphi / \beta)^{1 / 2}$, as in the case $\alpha=0$, the other $r$ solutions are in the curve centered near the origin.

When $\zeta \ll 1$ the two closed curves are actually ovals; increasing $\alpha$ the two ovals approach each other assuming the form of two beans, then they deform in two concentric ovals, one inside the other, which contain the origin of the coordinates (Belfiore and Caputo, 1996).

$\alpha=0$ physically means that the induction is due to the polarization charges and, in the relation $D=P+E$, where $P$ is the part of the field which is due to the polarization of the charges, the term $E$ is neglected.

When $z$ is real but not rational eq. (7.15) is not rigorously valid but considered an approximation; when $z$ is not a rational setting

$$
x=\rho e^{i \vartheta}
$$

we may write eq. (7.15)

$$
\begin{gathered}
\alpha \rho^{2+z} e^{i(\theta+2 l \pi)(2+z)}+\beta \rho^{2} e^{2 i(\theta+2 l \pi)}+ \\
+\varsigma \rho^{z} e^{i(\theta+2 l \pi) z}+\varphi=0
\end{gathered}
$$

with $k= \pm 1, \pm 2 \pm 3 \pm 4 \ldots . . \pm n \ldots$ and, separating the real and imaginary parts of (7.18), we obtain

$$
\begin{aligned}
& a \rho^{2+z} \cos (\theta(2+\theta)+2 k \pi)+d \rho^{2} \cos 2 \theta+ \\
& \quad+b \rho^{z} \cos (\theta+2 k \pi) z+c=0 \\
& a \rho^{2+z} \sin (\theta(2+\theta)+2 k \pi)+d \rho^{2} \sin 2 \theta+ \\
& \quad+b \rho^{z} \sin (\theta+2 k \pi) z=0 .
\end{aligned}
$$

Since there is a solution for each value of $l$ we obtain an infinite number of solutions.

The increase in $r$ implies that also $n$ increases because $r<n$. It is verified, in all the cases which we have discussed numerically (Belfiore and Caputo, 1996), that increasing $n$ and $r$ the three curves, where the poles are located, have centers in $\pm(\varphi / \beta)^{1 / 2}$ and in the origin of the coordinates; however this property has been verified only in the computer cases considered and has not been mathematically proved.

\section{The fatigue of anelastic and dielectric media}

\subsection{Introduction. The phenomenological model}

In a 1966 note Caputo suggested the following 1D constitutive stress $(\tau)$-strain $(\varepsilon)$ relation

$$
\tau=\mu \varepsilon+\eta \partial^{z} \varepsilon / \partial t^{z} \quad 0<z<1
$$

which implies an almost frequency independent $1 / Q$ and which was used later (Caputo, 1976) to represent the phenomenon of fatigue of anelastic media.

Equation (8.1) leads to the following equilibrium equation

$$
\rho \partial^{2} u / \partial t^{2}=\left(\mu+\eta \partial^{z} / \partial t^{z}\right) \partial^{2} u / \partial x^{2}
$$




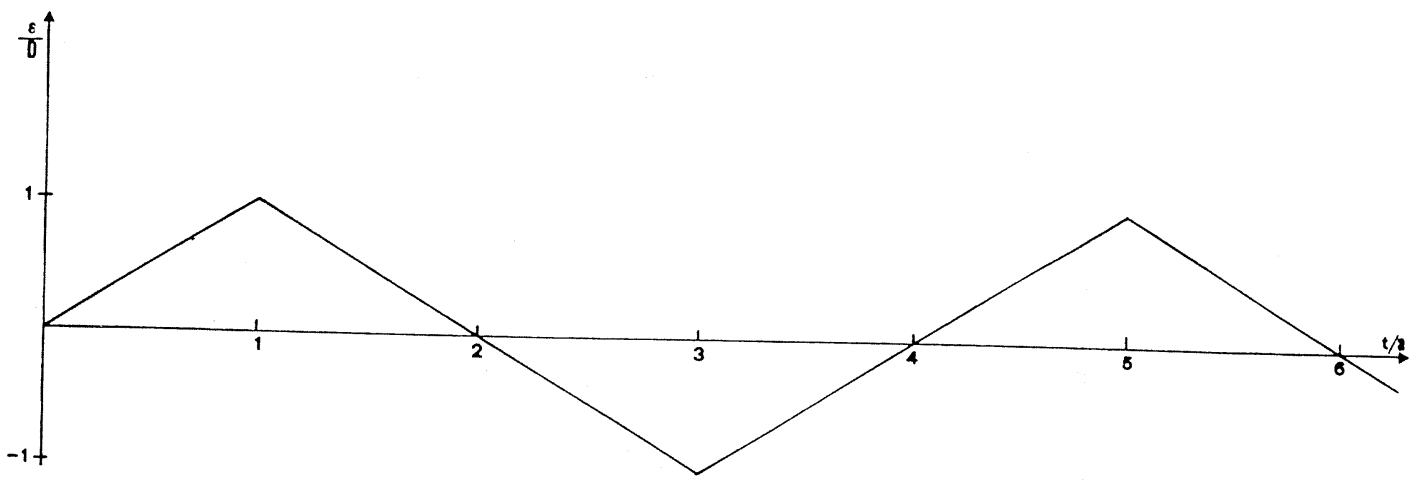

Fig. 3. Saw teeth function used for the computation of the hysteresis loops of figs. 4 and 5 .

Table I. Analytic representation of the term in square brackets of (8.4).

\begin{tabular}{|c|c|c|c|c|}
\hline & $\tau$ & $\epsilon$ & $t$ & $(1-z) !\left(d^{z} \in / d t^{z}\right)$ \\
\hline & 0 & $0, \quad 1$ & 0,1 & $t^{1-z}$ \\
\hline \multirow[t]{2}{*}{$n=0$} & 1 & $1,-1$ & 1,3 & $t^{1-z}-2(t-1)^{1-z}$ \\
\hline & 2 & $-1, \quad 1$ & 3,5 & $t^{1-z}-2(t-1)^{1-z}+2(t-3)^{1-z}$ \\
\hline \multirow[t]{2}{*}{$n=1$} & 3 & $1,-1$ & 5,7 & $t^{1-z}-2(t-1)^{1-z}+2(t-3)^{1-z}-2(t-5)^{1-z}$ \\
\hline & 4 & $-1, \quad 1$ & 7,9 & $t^{1-z}-2(t-1)^{1-z}+2(t-3)^{1-z}-2(t-5)^{1-z}+2(t-z)^{1-z}$ \\
\hline
\end{tabular}

where $u$ is the displacement and $\rho$ the density of the medium.

In the 1976 note Caputo discussed the case $\mu=0$ which leads to the generalized diffusion equation. In fact (8.2) may be written

$$
(\rho / \eta) \partial^{v} u / \partial t^{v}=\partial^{2} u / \partial x^{2}
$$

with $v=z+2$.

The study of the time domain form eq. (8.3) was made by Caputo (1976), in the case when $0<z<1$, and more extensively by Wyss (1986), Schneider and Wyss (1989) and Mainardi (1994) who also gave the Green function in different forms for $-2<z<0$; the formulation of the Green function given by $\mathrm{Ca}$ puto (1996a) for the study of porous media, in our opinion, is very simple ad practical.

To see how eq. (8.1) leads to fatigue let us consider an input signal $\varepsilon=f(t)$ with the form of the periodic saw tooth with period $4 a$ and amplitude $D$ shown in fig. 3. The stress response of the medium is

$$
\begin{array}{r}
\tau=\mu f(t)+(\eta D / a(1-z) !) \\
{\left[t^{1-z}-2 \sum_{j}(-1)^{j}(t+a-2 j a)^{1-z}\right] .}
\end{array}
$$

Neglecting the first term of the right hand member of (8.4), which gives no contribution to anelastic phenomena, for the first two and a half cycles, the analytic expression of eq. (8.4) is specified in table I and represented in figs. 4 and 5 .

In figs. 4 and 5 it is clear that the successive loops are not overlapping, and that an increasing modules of the stress is associated with the successive deformations $\varepsilon= \pm D$. 


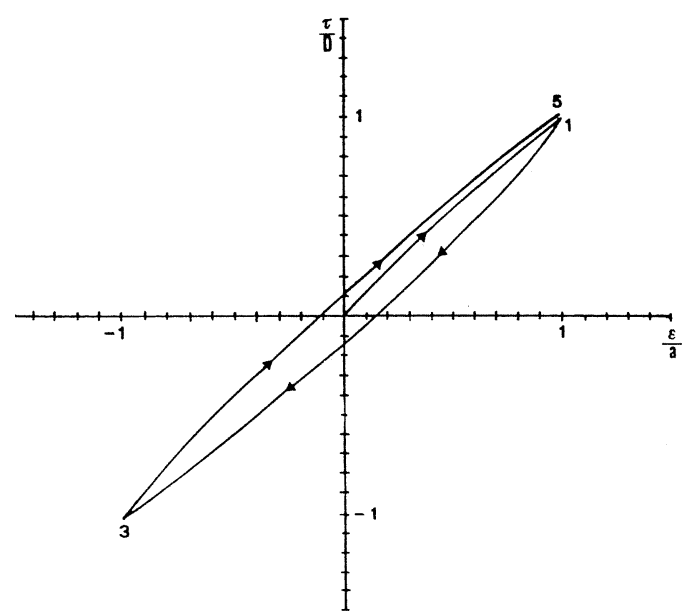

Fig. 4. Hysteresis loop response to the saw teeth input shown in fig. $3, z=0.1$ is the order of differentiation.

The successive increases in the stress $\sum_{n} F(n)$, between the $n$-th and the $n+1$ cycle, when $\varepsilon= \pm D$ are expressed by

$$
\begin{gathered}
F(n)=(\eta D / a(1-z) !)\left[(4 n+5)^{1-z}-(4 n+1)^{1-z}-\right. \\
\left.-2(4 n+4)^{1-z}+2(4 n+2)^{1-z}\right]
\end{gathered}
$$

which gives also the first increase for $n=0$.

An estimate of the sum of the series made with the terms defined by eq. (8.5) can be made considering $n$ as a continuous variable ad integrating between zero and infinity, we obtain

$$
\begin{gathered}
(D \eta / a) \varphi(z)= \\
=(D \eta / a)\left[1-5^{2-z}+2\left(4^{2-z}-2^{2-z}\right)\right] / 4(2-z) !
\end{gathered}
$$

which is positive and limited for $0<z<1$ and plotted in fig. 6.

If there is a value $n^{*}$ of $n$ for which $\tau$ exceeds the yield stress of the material, this value indicates the limit of the resistance of the material to the fatigue of the vibrations. This limit $n^{*}$ may be computed by considering it the un-

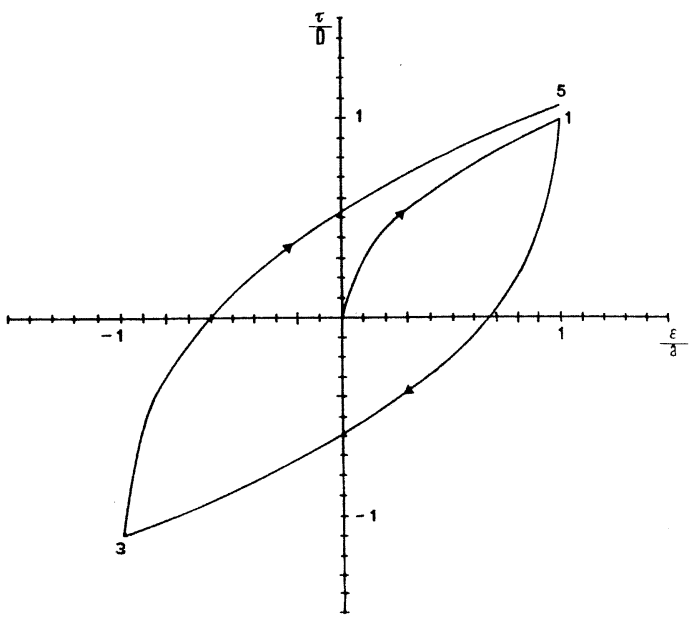

Fig. 5. Hysteresis loop response to the saw teeth input shown in fig. $3, z=0.5$ is the order of differentiation.

known upper limit of the integral mentioned above; in fact the sum of the successive $F(n)$, for $n=0,1,2, \ldots . ., n^{*}$, would give the observed yield point.

The value $n^{*}$ corresponds to the time $t^{*}=4 a n^{*}$ with $4 a$ period of the vibration, when yield would occur.

Of course it is quite possible that, for a given observed breaking strength, the value of $n^{*}$ does not exist because the integral is limited for $n \rightarrow \infty$ and the limit is smaller than the value of the stress giving fatigue for the assumed deformation $D$. The computation of the

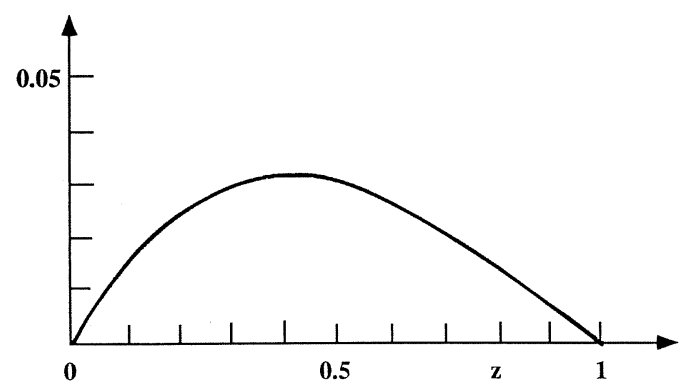

Fig. 6. The function $\varphi(z)$ from formula (8.6). 
hysteresis loop for $\varepsilon=\sin \omega t$ (instead of $\varepsilon$ as the saw teeth shown in fig. 3) would bring us to the same conclusion considering the fact that the loop does not close itself at $\varepsilon=D$ since the stress increases at the successive passages at $\varepsilon=D$.

One may estimate the sum of the first $n+1$ terms using the power series expansion of (8.5) cut after the fourth order derivative. We assume that $4 n+3$ is the initial point, \pm 2 and \pm 1 are increments and cut the series after the fourth order derivatives; since even order derivatives cancel out in each of the differences and the first order derivatives cancel out because off the double difference of the terms, we obtain

$$
\begin{aligned}
& (D \eta / a(1-z) !) \int_{n}^{\infty}\left[(4 n+5)^{1-z}-(4 n+1)^{1-z}\right]- \\
& \left.\quad-2(4 n+4)^{1-z}+2(4 n+2)^{1-z}\right] d n= \\
& =-(D \eta / a(1-z) !) z(z-1)(4 n+3)^{-1-z} / 2= \\
& =-(D \eta / a(-1-z) !)(4 n+3)^{-1-z} / 2 .
\end{aligned}
$$

The additional stress $r^{* *}$ built in the first $n+1$ cycles is then

$$
\begin{aligned}
& {[(D \eta / a) \varphi(z)-(D \eta / a) \psi(z, n) /(2(-1-z) !)]} \\
& \psi(z, n)=(4 n+3)^{-1-z} .
\end{aligned}
$$

Obviously, in general, in order for the additional stress (8.8) to be significant to cause fatigue it must be relevant relative to $\mu D$.

The total stress $\tau^{* *}$, after the first $n+1$ cycles, is then

$$
\begin{array}{cc}
\tau^{* *}=(D \eta / a)[\varphi(z)+\psi(n)(2(-1-z) !)] & +\mu D, \\
& n>0 \\
\tau * *=(D \eta / a) \varphi_{0}(z)+\mu D, & n=0 \\
\varphi_{0}(z)=F(0)=(D \eta / a)\left(-1+5^{1-z}-\right. & \\
\left.-2\left(4^{1-z}-2^{1-z}\right)\right) /(1-z !) . &
\end{array}
$$

Let the yield point of the material be $\tau^{*}$. If $\tau^{*}<\tau^{* *}(z, \infty)$ then eq. (8.9) allows to estimate the value of $n^{*}$ as function of $a, \tau, D$ and $\mu / \eta$, where, when $\omega^{z}(\eta / \mu) \sin \left(\pi_{z} / 2\right) \ll 1$, $\mu / \eta /$ may be computed from the equation

$$
1 / Q=\omega^{z}(\eta / \mu) \sin (\pi z / 2)
$$

obtained by Caputo (1976) for the case when $\omega^{z} \eta \sin (\pi z / 2) \ll \mu$.

The value of $n^{*}$, the number of cycles giving fatigue, is obtained substituting $\tau^{* *}$ with $\tau^{*}$ in eq. (8.8) and considering that $\psi(z, n)$, defined in (8.8), is a decreasing function of $n$ for the values of $z$ assumed here $(0<z<1)$; setting

$$
\tau * a / \eta D=\alpha
$$

we see that when $\alpha<\varphi_{0}(z)$, the value of $n^{*}$ is zero (in which case the fatigue would occur in the first cycle); when

$$
\begin{aligned}
& \varphi(z)+\psi(z, 0) / 2(-1-z) !<\tau * a / \eta D-\mu a / \eta \\
& <\varphi(z)+\psi(z, \infty) / 2(-1-z) !=\varphi(z)
\end{aligned}
$$

the value of $n^{*}$ is positive and there will be fatigue after $n^{*}$ cycles have elapsed. $n^{*}$ is obtained from (8.9)

$$
\begin{gathered}
n^{*}=(1 / 4)\{[-2(-1-z) ! \\
\left.\left.\left[\left(\tau^{*}-\mu D\right) a / \eta D+\varphi(z)\right]\right]^{-1 /(1+z)}-3\right\} .
\end{gathered}
$$

The value of $n^{*}$ is very sensitive to small variations of $x=\alpha-\mu \alpha / \eta$. In fact, differentiating $n^{*}$ we find

$$
d n^{*} / d x=-\left(4 n^{*}+3\right) / 2(-1-z) !(x-\varphi(z)) 4(1+z)
$$

and, since $\varphi(z)-x$, near the point of fatigue, is very small, then a small variation of its values may cause large errors in the estimate of $n^{*}$.

Formula (8.12) may be written

$$
-\psi(z, 0) / 2(-1-z) !+\varphi(z)+\mu \alpha / \eta<\alpha<\varphi(z)+\mu \alpha / \eta
$$


or, after a few formal changes

$\left[D_{m}^{-1}+\tau * \alpha \psi(z, 0) / 2 \eta(-1-z) !\right]^{-1}>D>D_{m}$

$D_{m}^{-1}=\eta\left[\varphi(z)+\mu / \tau^{*}\right] / \tau^{*} a$

which sets the limit to $D$ in order to have fatigue after the first cycle. If $D<D_{m}$ then fatigue occurs during the first cycle.

For the estimate of $n^{*}$ from eq. (8.13) we write it as follows

$n^{*}=(1 / 4)\left\{\left[A(z) a\left(\tau^{*}-\mu D\right) / \eta D+B(z)\right]^{-1 /(1+z)}-3\right\}$

$A=[-2(-1-z) !]>0$,

$B=[-2(-1-z) !] \varphi(z)>0$.

With algebraic considerations we obtain from (8.17) limitations on $D$ similar to those expressed by (8.16). In fact since $A>0$ and $B>0$ formula (8.17) implies that in order to have $n^{*}$ real and positive it must be

$0<-A a\left(\tau^{*}-\mu D\right) / \eta D+B<3^{-1-z}$

or, since $A>0$,

$\varphi(z)>a\left(\tau^{*}-\mu D\right) / \eta D>\varphi(z)-3^{-1-z} / A$

which may be more simply written

$$
0.02 \eta / a>\tau * / D-\mu>0.0035 \eta / a
$$

since $\varphi(z)-3^{-1-z} / A$, as well as $\varphi(z)$, are positive functions of $z[0,1]$ with lower limit 0 for $z=0$ and $z=1 ; \varphi(z)-3^{-1-z} / A$, has upper limit 0.0035 near $z=0.5$ and $\varphi(z)$ has upper limit 0.033 near $z=0.5$. The function $\varphi(z)-3^{-1-z} / A$, is shown in fig. 7 .

Formula (8.19) is more explicit than (8.11).

When a constant stress $\tau_{1}$ is applied to the material in addition to the cyclic one and $\tau^{*}$ is the yield stress, the number of cycles $n^{*}$ giving fatigue is

$$
\begin{aligned}
& n^{*}=0.25\left\{\left[A\left(\tau_{2}+\tau_{1}-\mu D\right) a / \eta D+B\right]^{-1 /(1+z)}-3\right\} \\
& \tau_{2}=\tau^{*}-\tau_{1}
\end{aligned}
$$

where we obviously verify that, when the static constant stress $\tau_{1}$ is larger, then the stress needed to reach the yield point is smaller and therefore fatigue will be reached in a smaller number of cycles.

Experimental data on the phenomenon of fatigue of anelastic materials as a function of the deformation, or of the frequency or of the temperature are very scarse in the linear field. Only recently have some interesting data become available (Yoshida et al,. 1978) which have features which may be tentatively interpreted in the linear field and allow some checks of formula (8.19). Also, an analysis more detailed than that appeared in the paper by Yoshida et al. (1978) allows some interesting considerations.

The values of strain in all the experiments of Yoshida et al. (1978) are very high and the linear stress is mostly less than $40 \%$ of the total stress, therefore we doubt that a quantitative test to our model may be significantly made with these data; the model may be tested only from a qualitative point of view.

We may say that, inspite of the fact that the linear stress in the experiments of Yoshida

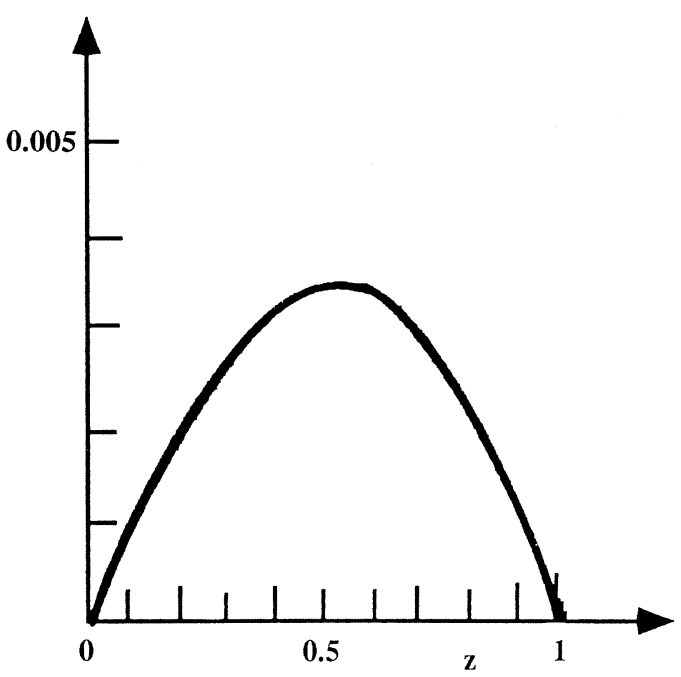

Fig. 7. The function $\left[\varphi(z)-3^{-1-z /(-2(-1-z) !)]}\right.$ appearing in (8.19), where $\varphi(z)$ is defined in (8.6) and shown in fig. 6 . The ordinate is in units of $D / a$. 


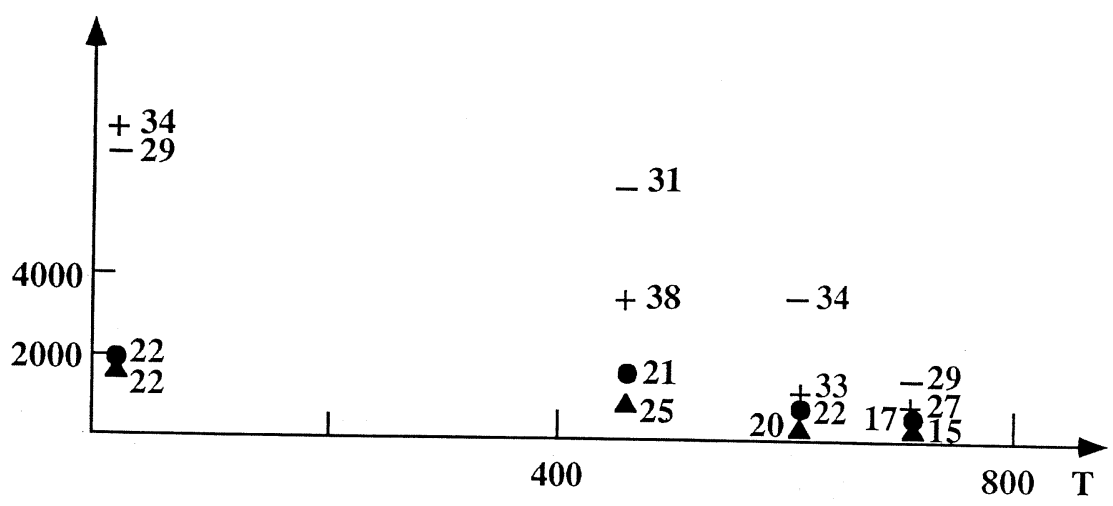

Fig. 8. Fatigue test data. The ordinate is the number of cycles to failure and $T$ is the temperature in degrees centigrades. The data are from Yoshida et al. (1978). The number near the symbols give the percent of linear strain. The material is Cr-10Ni-Ti-BIV steel. The meaning of the symbols in the figure is explained as
follows:

$\begin{array}{cccccccccc}D & f & a & D^{\prime} & & D & f & a & D^{\prime} & \\ 0.02 & 0 . \overline{6} & 1.5 & 2 / 150 & \bullet & 0.2 & 0 . \overline{6} & 1.5 & 2 / 15 & \bullet \\ 0.01 & 1 . \overline{3} & 0.75 & 2 / 150 & - & 0.1 & 1 . \overline{3} & 0.75 & 2 / 15 & - \\ 0.02 & 0.0 \overline{6} & 15 & 2 / 1500 & \boldsymbol{\Delta} & 0.2 & 0.00 \overline{6} & 150 & 2 / 1500 & \boldsymbol{\Delta} \\ 0.01 & 0.1 \overline{3} & 7.5 & 2 / 1500 & + & 0.1 & 0.01 \overline{3} & 75 & 2 / 1500 & + \\ & & & T=20^{\circ} \mathrm{C}, \quad T=450^{\circ} \mathrm{C}, \quad T=600^{\circ} \mathrm{C}, & T=700^{\circ} \mathrm{C} & & \end{array}$

et al. (1978) is less than $40 \%$ of the total stress, most properties of the model presented here are verified by the data of Yoshida et al. (1978), which are rearranged and plotted in figs. 8,9,10, 11 and 12 for several values of the temperature and two values of the strain used in the experiments.

One may note that the number of cycles to failure is a decreasing function of $D$ (the solid circles are below the minuses and the triangles are below the pluses) and that the number of cycles to rupture increases with the elastic fraction of the total strain, that is, when the non linear and plastic phenomena decrease.

It is also worth noting that the percentual part of elastic strain is a decreasing function of the frequency and of the total strain.

In order to make further consideration on formula (8.21) (or (8.13)) let us rewrite (8.21) introducing the strain rate $D^{\prime}$

$$
\begin{aligned}
& n^{*}=0.25\left\{\left[-A\left(\tau_{2}+\tau_{1}-\mu D\right) / \eta D^{\prime}+B\right]^{-1 /(1+z)}-3\right\} \\
& \tau_{2}=\tau^{*}-\tau_{1}
\end{aligned}
$$

from which it follows that $n^{*}$ is a decreasing function of $D^{\prime}$.

However this property of the model, which was theoretically shown by Caputo (1979), is not verified in the data of Yoshida et al. (1978); the cause may be that the strain, in the experimental data it is too large to be considered in the linear field and, according to Yoshida et al. (1978), most of the strain is plastic; therefore we could hardly expect that a combination of non linear and plastic phenomena be represented by a linear model in all its features.

However the decrease of $n^{*}$ with increasing $D^{\prime}$ is verified in the laboratory experiments on cyclic loads applied to rocks made by Scholz and Koczinsky (1979). In fact these authors found that the number of cycles to fatigue is a function decreasing with the amplitude and with the rate of the applied cyclic stress, in agreement with the theoretical results of Caputo (1979) and the formulae obtained here. 


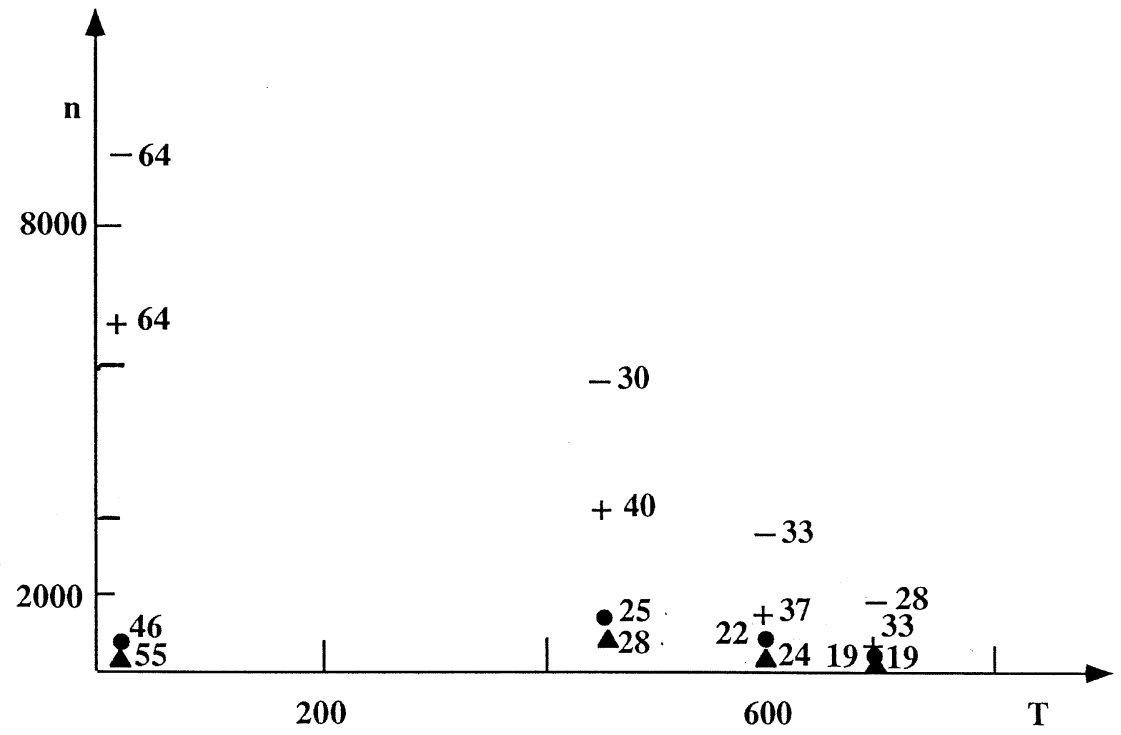

Fig. 9. As in fig. 8 for $18 \mathrm{Cr}-10 \mathrm{Ni}-\mathrm{Ti}-\mathrm{CII}$ steel from the data of Yoshida et al. (1978).

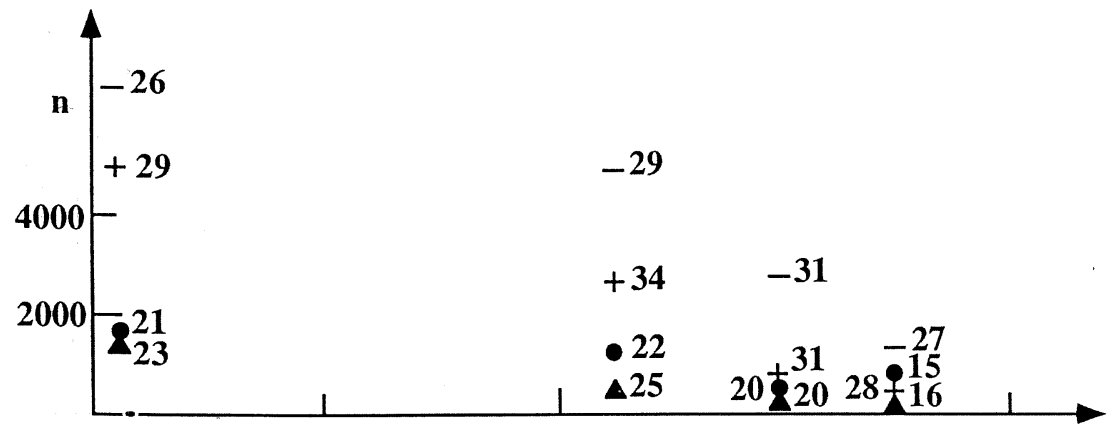

Fig. 10. As in fig. 8 for $18 \mathrm{Cr}-10 \mathrm{Ni}-\mathrm{Ti}-\mathrm{BII}$ steel from the data of Yoshida et al. (1978).

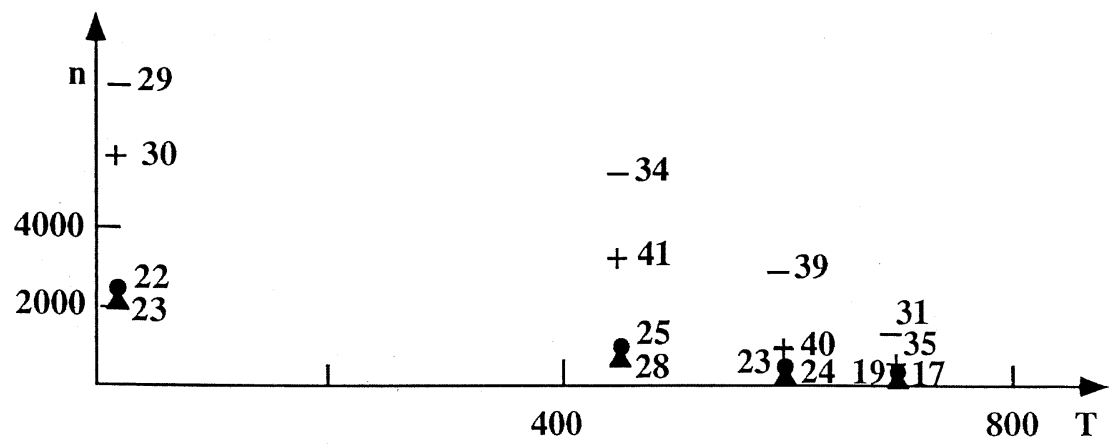

Fig. 11. As in fig. 8 for $18 \mathrm{Cr}-10 \mathrm{Ni}-\mathrm{Ti}-\mathrm{BIII}$ steel from the data of Yoshida et al. (1978). 


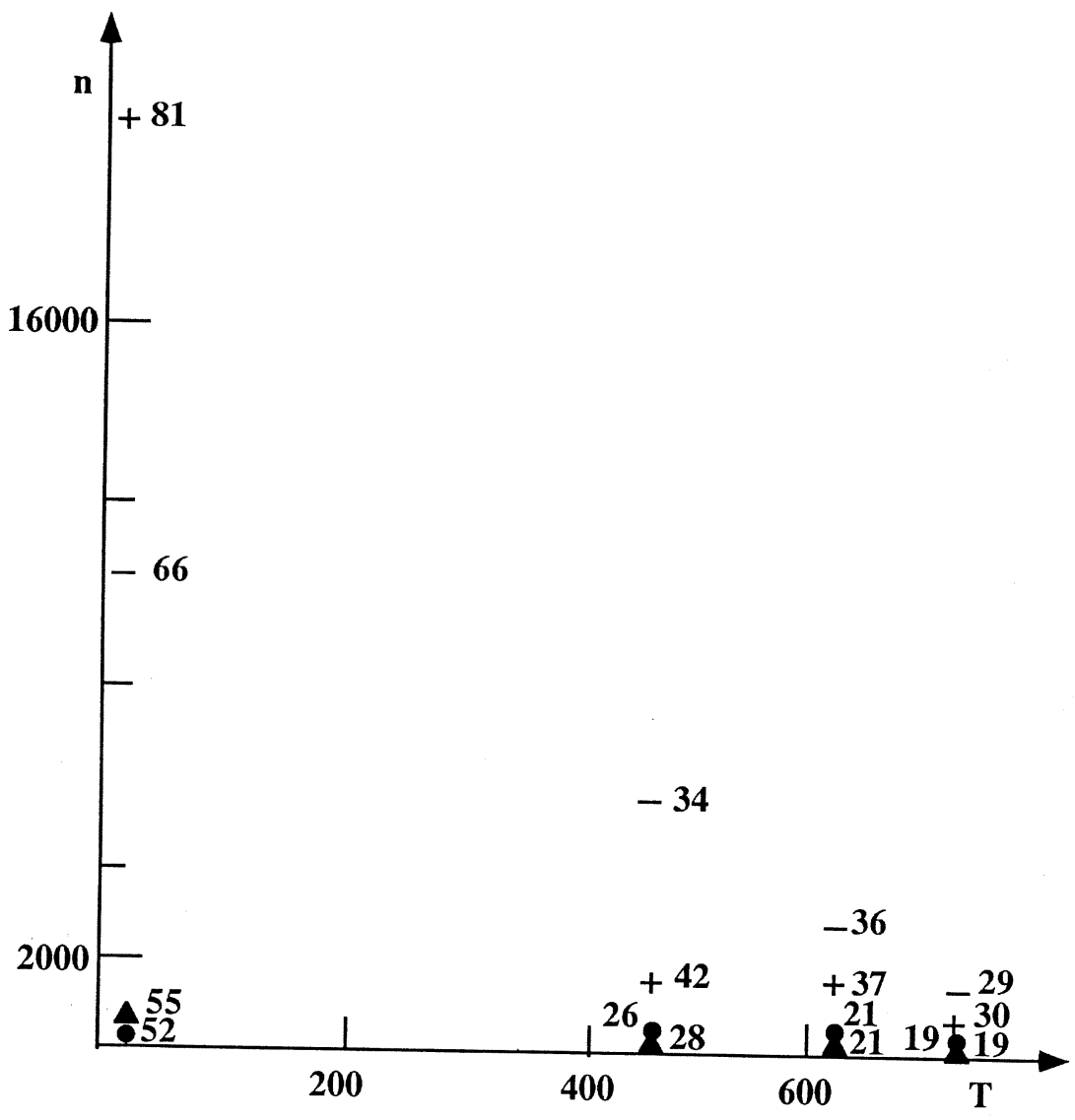

Fig. 12. As in fig. 8 for $18 \mathrm{Cr}-10 \mathrm{Ni}-\mathrm{Ti}-\mathrm{CI}$ steel from the data of Yoshida et al. (1978).

\subsection{The use of the general stress-strain relations}

A somewhat more general study of hysteresis may be made with the same methodology used but assuming that the stress-strain relation is of the more general type, already considered in section 2 ,

$$
\left(\sigma+\eta \partial^{z} / \partial t^{z}\right) \tau=\left(\alpha+\gamma \partial^{z} / \partial t^{z}\right) \varepsilon
$$

whose LT gives

$T=(\gamma / \eta)\left[1+(-\sigma / \eta+\alpha / \gamma) /\left(\sigma / \eta+p^{z}\right)\right] E$
In order to obtain $\tau$ from (8.24) we must deconvolve it; we find (Caputo, 1984)

$$
\begin{aligned}
& \tau=(\sigma / \eta) f(t)+f(t) *(-\sigma / \eta+\alpha / \gamma) \chi(t) \\
& \chi(t)=((\sin \pi z) / \pi z) t_{4}^{-1} \int_{0}^{\infty} u^{1 / z} \\
& \left(\exp \left(-u^{1 / z} t / t_{4}\right)\right) d u /\left(u^{2}+2 u \cos \pi z+1\right)
\end{aligned}
$$

$t_{4}=(\eta / \sigma)^{1 / z}$

where $t_{4}$ is the relaxation time and $f(t)$ is the input strain.

A case of geophysical interest, as shown by Körnig and Müller (1989), is that when in the stress-strain relation (8.23) it is assumed $\alpha=0$. 
In order to simplify the computations, let us assume also that $\varepsilon=f(t)=D \sin (\pi t / 2 a)$ we obtain from $(8.25)$

$$
\tau=(\gamma / \eta)[\delta(t)-\chi(t)]^{*} f(t) .
$$

In order to estimate the total increase in the modules of the stress between two successive points at the times $t_{2}=4 m a$ and $t_{3}=4(m+1) a$ respectively, that is in the $m$-th cycle, due to the memory term in (8.26), we first compute the convolution in (8.26)

$D t_{4}^{-1} \int_{0}^{\infty}\left(\exp \left(-u^{1 / z} t / t_{4}\right)\right)\left[\left(u^{1 / z} / t_{4}\right) \sin (\pi / 2 a) t+\right.$

$\left.-(\pi / 2 a) \cos (\pi / 2 a) t+(\pi / 2 a) \exp \left(-u^{1 / z} t / t_{4}\right)\right] d u /$

$\left[(\pi / 2 a)^{2}+\left(u^{1 / z} / t_{4}\right)^{2}\right]\left(u^{2}+2 u \cos \pi z+1\right)$.

Assuming in (8.27) $t=4 m a$ and $t=4(m+1) a$ and then subtracting the two expressions obtained we find the increase in the modules of the stress in the $m$-th cycle

$\Delta \tau_{m}=t_{4}^{-1} H(\pi / 2 a)(\sin z \pi / z \pi) \int_{0}^{\infty} u^{1 / z}(1+$

$\left.-\exp \left(-u^{1 / z} 4 a / t_{4}\right)\right)\left[\exp \left(-u^{1 / z} 4 m a / t_{4}\right)\right]$

$d u /\left[(\pi / 2 a)^{2}+\left(u^{1 / z} / t_{4}\right)^{2}\right]\left(u^{2}+2 u \cos \pi z+1\right)$

$H=D \gamma / \eta$

which is decreasing to zero with increasing $m$

Formula (8.28) may be used for the estimate of the number of cycles $m^{*}$ which gives fatigue. For this purpose one may follow the same procedure used in the previous paragraph when the stress-strain relation was of the type (8.1), which is a special case of (8.23) with $\eta=0$ and $\sigma=1$, and the strain relation was of the type (8.1), which is a special case of (8.23) with $\eta=0$ and $\sigma=1$, and the applied strain had the shape of a set of saw teeth; in the case considered now however, it is not possible to render explicit $m^{*}$ and one should proceed with numerical methods as follows.

The total increase in the modules of the stress in an infinite number of cycles is obtained assuming $m$ as a continuous variable and integrating (8.28) with respect to $m$ from zero to infinity; we find

$$
\begin{aligned}
& \Delta \tau=H \Phi(z) / 2 \pi \\
& \Phi\left(z, 4 a / t_{4}\right)=\Psi_{1}\left(z, 4 a / t_{4}\right)-\Psi_{2}\left(z, 4 a / t_{4}\right) \\
& \Psi_{1}\left(z, 4 a / t_{4}\right)=(\sin \pi z / \pi z) \int_{0}^{\infty} d u / \\
& \quad\left[1+\left(\left(4 a / t_{4}\right) u^{1 / z} / 2 \pi\right)^{2}\right]\left(u^{2}+2 u \cos \pi z+1\right) \\
& \Psi_{2}\left(z, 4 a / t_{4}\right)=(\sin \pi z / \pi z) \int_{0}^{\infty} \exp \\
& \quad\left(-u^{1 / z} 4 a / t_{4}\right) d u /\left[1+\left(\left(4 a / t_{4}\right) u^{1 / z} / 2 \pi\right)^{2}\right]\left(u^{2}+\right. \\
& \quad+2 u \cos \pi z+1)
\end{aligned}
$$

In fig. 13 the values of $\Phi\left(z, 4 a / t_{4}\right)$ are shown for several values of $z$, as a function of the period $4 a$ of the input signal measured in units of relaxation time $t_{4}$.

As a geophysical application of the theory developed we tentatively estimate the fatigue accumulated by the tidal deformation caused by the Sun and the Moon in the mantle of the Earth.

Concerning the Earth's mantle the discussion may only be tentative because we have for $\eta / \sigma$ and $z$ only average values over the very wide range of temperatures of the mantle, which does not fit the theory developed here for homogeneous media. Only tentative conclusions may be obtained.

The task could be somewhat easier for the crust because the temperature variations with depth are more limited, but the reliable data concerning the crust are even more scarse and unreliable than those of the mantle.

The values of parameters describing the dispersion and the dissipation in the Earth's mantle have been tentatively determined, for the stress-strain relation of the type (8.23) and assuming $\alpha=0$, as suggested by the results of the analysis of the rheology of the mantle made by Körnig and Müller (1989).

From the analysis of the Earth's tide data of Slichter et al. (1964) and of the $Q$ of the free torsional modes of Smith (1961), Alsop et al. (1961), McDonald and Ness (1961), Bozzi Zadro and Caputo (1968), the values of the pa- 


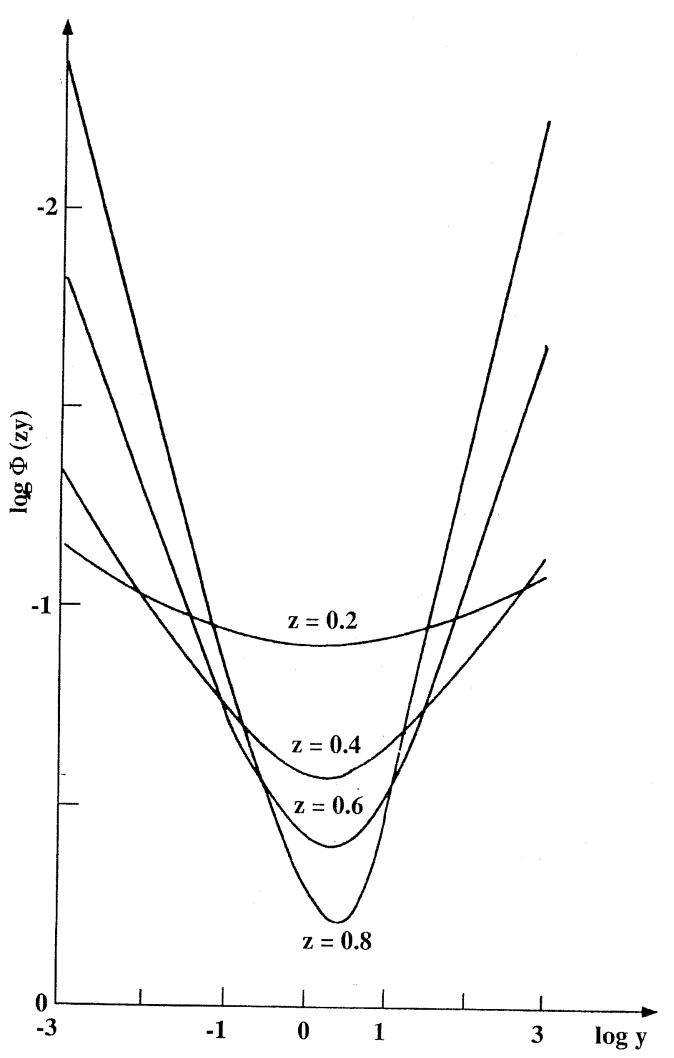

Fig. 13. The function $\Phi\left(z, 4 a / t_{4}\right)=\Psi_{1}\left(z, 4 a / t_{4}\right)-$ - $\Psi_{2}\left(z, 4 a / t_{4}\right)$ for several values of $z$. In the abscissa $y$ is in units of $4 a / t_{4}$.

rameters appearing in $(8.25)$ have been tentatively estimated (Caputo, 1990); they are

$$
\eta / \sigma=3370 \mathrm{~s}^{1 / z}, z=0.6, t_{4}=(\eta / \sigma)^{1 / z}=757500 \mathrm{~s}
$$

and it is also assumed that in the mantle the rigidity is $\mu=\gamma / \eta=10^{5} \mathrm{MPa}$.

The values of $\Phi\left(z, 4 a / t_{1}\right)$ for the 24 and $12 \mathrm{~h}$ tidal periods are $\Phi(0.6,24 / 210)=0.194$ and $\Phi(0.6,12 / 210)=0.140$ respectively, where 210 is the relaxation time, $t_{4}=757500 \mathrm{~s}$, measured in hours.

Assuming that the deformations of the 24 and $12 \mathrm{~h}$ tidal components are at most $510^{-8}$ each, which is close to reality, then
$H / 2 \pi=810^{2} \mathrm{~Pa}$ in (8.29) and one may see from (8.29) that the tidal deformations of the mantle due to the Sun and the Moon may cause a stress increase in at most $10 \mathrm{~Pa}$, which seems a negligible contribution to the stress distribution in the Earth's mantle.

In the Earth's crust one may see that the Earth's tide data tentatively give $H / 2 \pi=$ $=2.5 \cdot 10^{2} \mathrm{~Pa}, t_{4}=300$ years, $z=0.6$ and $\mu=$ $=3 \cdot 10^{4} \mathrm{MPa}$ (Caputo, 1993); applying formula (8.29) one may see that the stress accumulated is even smaller than that accumulated in the mantle and that Earth's tides may not be considered a good candidate phenomenon for the release of the elastic energy accumulated in the crust by the tectonic activity.

However it is important to note that, since laboratory experiments show that the yield stress is decreasing with increasing temperature which in turn is increasing significantly with depth, the problem of the triggering of earthquakes by fatigue should be revisited taking into account the effect of the temperature.

As a final note, we suggest that the phenomenon of fatigue in anelastic media, phenomenologically and physically discussed in this section, may be discussed in the same manner also for dielectric media.

\section{REFERENCES}

Alsop, L.E., G.H. SutTon and M. EwING (1961): Free oscillations of the Earth observed on strain and pendulum seismographs, J. Geophys. Res., 66(2), 631-641.

BAGLEY, R.L. and P.J. ToRvIK (1983a): A theoretical basis for the application of fractional calculus to viscoelasticty, J. Rheology, 27(3), 201-210.

BAgley, R.L. and P.J. TorviK (1983b): Fractional calculus - A different approach to the analysis of a viscoelastically damped structure, A.I.A.A., 25(5), 741-748.

BAGLEY, R.L. and P.J. TorvIK (1986): On the fractional calculus models for viscoelastic behaviour, J. Rheology, 30 (1), 133-155.

Belfiore, L. and M. CAPuto (1996): The splitting of the free modes caused by the rational power of the imaginary frequency (in preparation).

Bossi ZADro, M. and M. CAPUTO (1968): Spectral, bispectral analysis and $Q$ of the free oscillations of the Earth, Nuovo Cimento, Suppl., 1 (6), 67-81.

CAPUTO, M. (1966): Linear models of dissipation whose $Q$ is almost frequency independent, Annali di Geofisica, 19 (4), 383-393.

CAPUTO, M. (1969): Elasticità e Dissipazione (Zanichelli, Bologna). 
CAPuto, M. (1976): Vibrations of an infinite plate with a frequency independent, Q, J. Acoust. Soc., Am., 60 (3), 634-639.

CAPUTO, M. (1979): A model for the fatigue in elastic materials with frequency independent, $Q, J$. Acoust. Soc. Am., 66 (1), 176-179.

CAPuTO, M. (1984): Relaxation and free modes of a selfgravitating planet, Geophys. J. R. Astron. Soc., 77, 789-808.

CAPUTO, M. (1986): Linear and non linear inverse rheologies of rocks, Tectonophysics, 122, 53-71.

CAPUTO, M. (1987): The interpretation of the $b$ and $b_{0}$ values and its implications on the regional deformations of the crust, Geophys. J. R. Astron. Soc., 90, 37-61.

Caputo, M. (1990): The splitting of the free torsional modes of the Earth caused by the rheology, Rend. Fis. Acc. Lincei, 9 (1), 119-125.

CAPuto, M. (1993a): Free modes, splitting and alterations of electrochemically polarizable media, Rend. Fis. Acc. Lincei, 9 (4), 89-98.

CAPuto, M. (1993b): The stress field in the crust and the sinking of mountains, J. Phys. Earth, 41, 109-126.

CAPUTO, M. (1994): A unified representation of the dispersion and of the dissipation in dielectric and anelastic media: consequences, in 14th International Congress of Int. Ass. Mathem. and Computer Simulation, Atlanta VA, USA, abstract, invited presentation.

CAPUTO, M. (1995a): Distance measurements, splitting of electromagnetic waves caused by the dispersion and GPS retrieval of the model atmosphere, Rend. Fis. Acc. Lincei, 9 (6), 19-36.

CAPUTO, M. (1995b): Distance measurements and splitting of electromagnetic waves in water caused by dispersion, Rend. Fis. Acc. Lincei, 9 (5), 103-113.

CAPUTO, M. (1995c): Mean fractional-order-derivatives differential equations and filters, in Annual Meeting American Mathematical Society, San Francisco, abstract, invited presentation.

CAPUTO, M. (1996a): The Green function of the diffusion in porous media with memory, Rend. Fis. Acc. Lincei, 9 (7), in press.

CAPUTO, M. (1996b): Rigourous time domain responses of polarizable media, Annali di Geofisica (in press).

CAPUTO, M. and F. MAINARDI (1971a): A new dissipation model based on a memory mechanism, Pure Appl. Geophys., 91, 134-147.

CAPUto, M. and F. MAINARd (1971b): Linear models of dissipation in anelastic solids, Il Nuovo Cimento, 1(2), 161-198.

Caputo, M. and W. Plastino (1996): Time domain fitting of polarizable media responses, in VI Workshop «di Elettromagnetismo», abstract.

CisotTi, V. (1911): L'ereditarietà lineare ed i fenomeni dispersivi, Il Nuovo Cimento, 6 (2), 234-244.

COLE, KS. and R.H. Cole (1941): Dispersion and absorption in dielectrics, J. Chem. Phys., 9, 341-349.
GRAFFI, D. (1936): Sopra alcuni fenomeni ereditari dell'elettrologia III, IV, V, Rend. Ist. Lomb. Sc. Lett. Arti, 2 (69), 124-181.

Hasted, J.B. (1973): Aqueous Dielectrics (Chapman and Hall).

HEAVISIDE, O. (1889): Electromagnetic Theory (The Electrician Printing and Publishing Co.), vol. II.

JACQUELIN, J. (1991): A number of models for CPA impedances of conductors and for relaxation in non Debye dielectrics, J. Non-Crist. Solids, 131, 1080-1083.

KNOPOFF, L. and G.J.F. MACDONALD (1958): Attenuation of small amplitude stress waves in solids, Rev. Mod. Phys., 30, 338-343.

KÖRNIG, H. and G. MüLlER (1989): Rheological models and interpretation of postglacial uplift, Geophys. J. R. Astron. Soc., 98, 245-253.

Liouville, J. (1832): Mémoire sur le calcul des différentielles à indices quelconque, J. Ecole Polytecnique, 13 (21), 71-162.

MAINARDI, F. (1994): On the initial value problem for the fractional diffusion wave equation, in Waves and Stability in Continuous Media, edited by S. RIONERO and T. RUGGERI (Singapore: World Scientific).

MCDonAlD, G.J.F. and N. Ness (1961): A study of the free oscillations of the Earth, J. Geophys. Res., 66, $1865-1911$

Patella, D., I. Pinto, M. Castricone and R. Di Maio (1987): Fitting discharges by Prony's method, Boll. Geofis. Teor. Appl., 29(113), 33-41.

Pelton, W.H., W.R. Sill and B.D. SMith (1983): Interpretation of complex resistivity and dielectric data, part I, Geophys. Trans., 29 (4), 297-330.

PeselniK, L., Hsi-Ping LiU and K.R. HARPer (1979): Observations of details of hysteresis loops in westerly granite, Geophys. Res. Lett., 6 (9), 693-696.

SCHNEIDER, W.R. and W. Wyss (1989): Fractional diffusion and wave equations, J. Math. Physics, 30, 134-144.

Scholz, C.H. and T.A. KoczInsKy (1979): Dilatancy anisotropy and the response of rock to large cycling loads, J. Geophys. Res., 84 (10), 5525-5534.

Slichter, L.B., G.J.F. MCDonAld, M. CAPUTO and C.L. HAGER (1964): Report of Earth tide results and of other gravity results at UCLA, Obs. Roy. Belgique, Comm. 236, Serie Geophys., 69, 124-130.

SMith, S. (1961): An investigation of the Earth's free oscillations, Thesis Calif. Inst. Techn., Pasadena.

Yoshida, S., K. Kanazawa, K. Yamagucji, M. Sato, U. KobAYSHA and N. SuZUKI (1978): Elevated temperature fatigue properties of engineering materials, Nat. Res. Inst. Metals, Tokyo, Japan.

Wyss, W. (1986): Fractional diffusion equation, J. Math. Phys., 27, 2782-2785.

(received May 10, 1996; accepted July 23, 1996) 